\title{
Phases and phase transitions in disordered quantum systems
}

\author{
Thomas Vojta \\ Department of Physics, Missouri University of Science and Technology, Rolla, MO 65409, USA
}

\begin{abstract}
These lecture notes give a pedagogical introduction to phase transitions in disordered quantum systems and to the exotic Griffiths phases induced in their vicinity. We first review some fundamental concepts in the physics of phase transitions. We then derive criteria governing under what conditions spatial disorder or randomness can change the properties of a phase transition. After introducing the strong-disorder renormalization group method, we discuss in detail some of the exotic phenomena arising at phase transitions in disordered quantum systems. These include infinite-randomness criticality, rare regions and quantum Griffiths singularities, as well as the smearing of phase transitions. We also present a number of experimental examples.
\end{abstract}

Keywords: phase transition, critical point, disorder, renormalization group, Griffiths singularity PACS: $64.60 .-\mathrm{i}, 75.10 . \mathrm{Nr}, 75.40 .-\mathrm{s}, 05.70 . \mathrm{Jk}, 05.50 .+\mathrm{q}$,

\section{OVERVIEW}

Phase transitions are fascinating phenomena that arise in a wide variety of physical, chemical, and biological systems. These range from the never-ending transformations of water between ice, liquid and vapor to transitions between different steady states in the dynamics of bio-populations. A particularly interesting class of transitions, the quantum phase transitions, occur when the quantum ground state of a material abruptly changes in response to a change in external conditions. Many realistic systems are not perfectly clean but contain impurities, defects, or other kinds of imperfections. The influence of such random disorder on phase transitions is therefore of prime interest, both from a conceptual point of view and for experimental applications.

This article gives an informal introduction into this topic at a level accessible for graduate students. It is based on the notes of a series of lectures that the author delivered at the XVII Training Course in the Physics of Strongly Correlated Systems in Vietri sul Mare, Italy in October 2012. The emphasis of the paper is on a pedagogical exposition of the fundamental ideas and methods rather than a comprehensive account of the literature. More details can be found in several recent review articles [1, 2, 3].

To make these lecture notes self-contained, we start with a brief summary of the fundamental concepts of classical and quantum phase transitions in Sec. 1. In Sec. 2 , we consider various types of disorder or randomness, and we derive criteria that govern under what conditions they can influence a phase transition. The strong-disorder renormalization group method is introduced in Sec. 3. Sections 4 and 5 are devoted to exploring some of the exotic phenomena arising at disordered quantum phase transitions, viz., Griffiths singularities and smeared transitions. Applications to magnetic quantum phase transitions in metals are discussed in Sec. 6. We conclude in Sec. 7. 


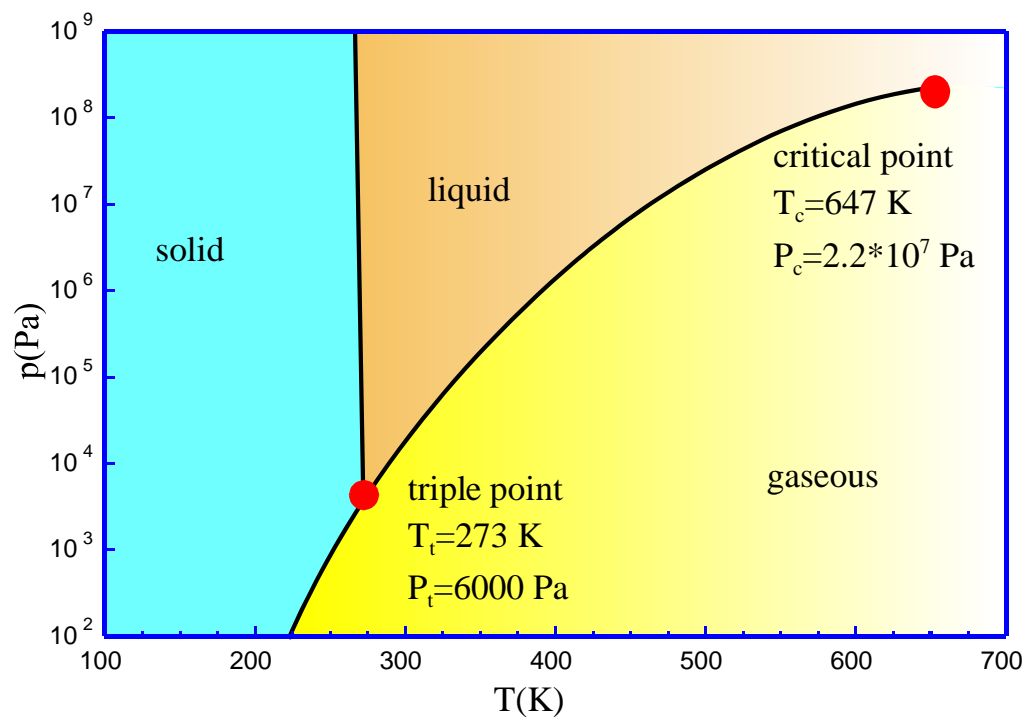

FIGURE 1. Phase diagram of water as a function of pressure $p$ and temperature $T$. The solid black lines mark first-order phase transitions. The liquid-gas phase boundary ends in a critical point at which the phase transition is continuous.

\section{PHASE TRANSITIONS AND QUANTUM PHASE TRANSITIONS}

In this section, we briefly collect some basic ideas about classical and quantum phase transitions to the extent required for the rest of the article. A reader unfamiliar with these topics will benefit from consulting text books such as Refs. [4, 5].

\subsection{Fundamental concepts}

\section{What is a phase transition?}

Consider, for example, the phase diagram of water reproduced in Figure 1. It shows in which phase (solid, liquid or gas) water is for given values of pressure $p$ and temperature $T$. If we cross one of the phase boundaries (marked by solid black lines), the properties of water change abruptly in response to a smooth change in pressure and/or temperature. This is the main characteristic of a phase transition. Formally, we can define a phase transition as a singularity in the free energy as function of the external parameters such as temperature, pressure, magnetic field, or chemical composition. ${ }^{1}$ A singular free energy also implies singularities in other thermodynamic quantities including internal energy, entropy, specific heat, and so on.

\footnotetext{
${ }^{1}$ A true singularity can only appear in the thermodynamic limit of infinite system size. For any finite system, the free energy is an analytic function because the partition function is a finite sum of exponentials.
} 


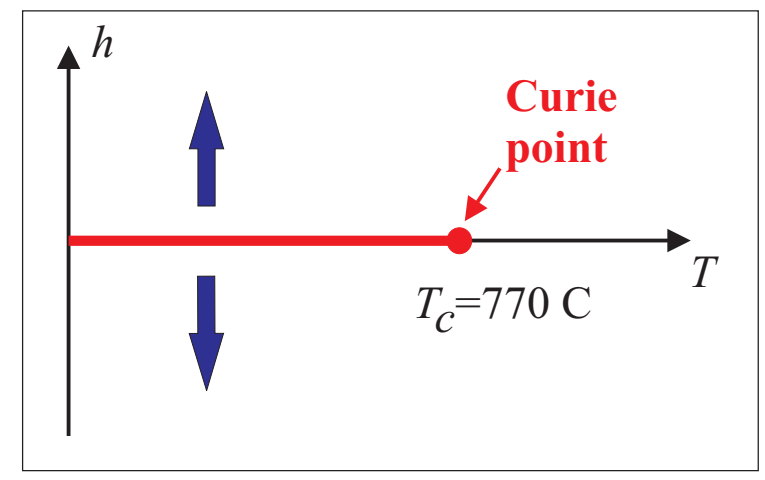

FIGURE 2. Schematic phase diagram of iron as a function of magnetic field $h$ and temperature $T$. The thick red line marks a first-order phase boundary between the spin-up and spin-down phases. It ends in a critical point (Curie point) at which the phase transition is continuous.

Phase transitions can be divided into two qualitatively different types or classes. To understand the difference, consider again the example of water. Imagine we heat up a piece of ice, starting at a temperature well below the melting point of $0^{\circ} \mathrm{C}$. Initially, the ice' temperature will rise smoothly as it absorbs more and more heat. However, after the temperature reaches $0^{\circ} \mathrm{C}$, the absorbed heat is used to melt the ice while the temperature is stuck at the melting point. Only after all ice has melted, the temperature resumes its rise. This means, the solid and liquid phases of water coexist at the phase transition point, and a nonzero amount of heat, the latent heat, is required to turn the solid phase of water into the liquid phase. Phase transitions following this scenario, namely phase coexistence and latent heat, are usually called first-order phase transitions. ${ }^{2}$

The phase transitions of water shown in Fig. 1 are all of first order, except for one single isolated point. To understand this, focus on the liquid-gas phase boundary. At ambient pressure (about $10^{5} \mathrm{~Pa}$ ), the properties of liquid water and water vapor are very different (for example, their densities differ by a factor of more than 1000). If we follow the phase boundary to higher pressures and temperatures, liquid and vapor become more and more similar until they become indistinguishable at $T_{c}=647 \mathrm{~K}$ and $p_{c}=2.21 \times 10^{7}$ $\mathrm{Pa}\left(374^{\circ} \mathrm{C}\right.$ and $\left.217 \mathrm{~atm}\right)$. This point is the so-called critical point. For $p>p_{c}$, water features only one fluid phase rather then two separate liquid and gas phases. Phase transitions occurring at such a critical point are called continuous phase transitions. ${ }^{3}$ Continuous phase transitions do not display phase coexistence because the two phases are indistinguishable at the transitions point. This implies that there is no latent heat.

Further prototypical examples of phase transitions can be found in a ferromagnet such as iron. Its magnetic phase diagram, shown in Fig. 2, is qualitatively very similar to the liquid-gas phase diagram of water. There is a line of first-order phase transitions

\footnotetext{
2 The name refers to Ehrenfest's classification [6] of phase transitions. At a first-order transition, a first derivative of the free energy is discontinuous.

${ }^{3}$ Ehrenfest further subdivided continuous transitions into second order transitions, third order transitions and so on according to which derivative of the free energy is discontinuous. Today, this subdivision is not used very much because there are few qualitative differences between the sub-classes.
} 


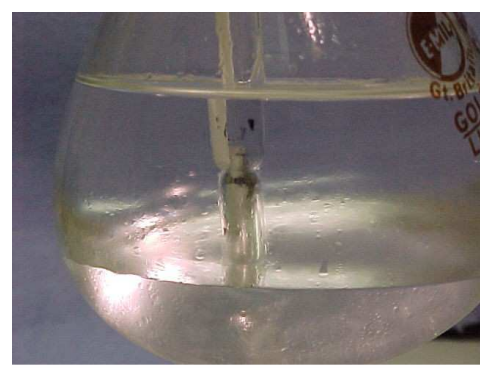

$18^{\circ} \mathrm{C}$

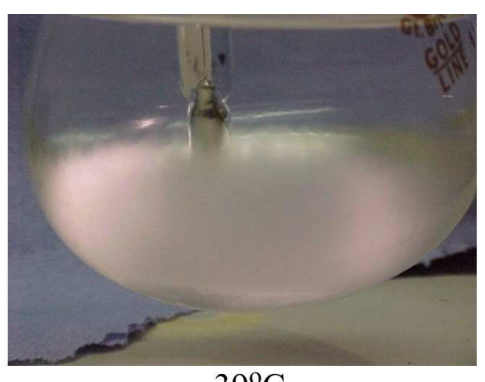

$39^{\circ} \mathrm{C}$

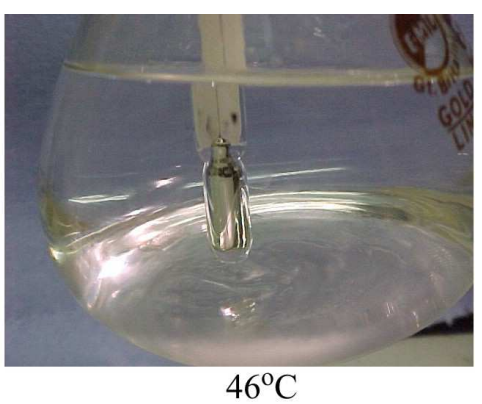

$46^{\circ} \mathrm{C}$

FIGURE 3. Critical opalescence near the mixing critical point of hexane and methanol (taken with permission from Ref. [8]).

at low temperatures and magnetic field $h=0$ that separates the spin-up and spin-down phases which coexist on the phase boundary. If we follow the phase boundary to higher temperatures, the magnetization decreases, thus the two phases become more similar. The phase boundary ends at the critical point (Curie point) at which the magnetization vanishes and the two phases (spin-up and spin-down) thus become indistinguishable.

\section{Critical behavior}

Critical points (continuous phase transitions) have many peculiar properties. One of the earliest and most striking manifestations of criticality was discovered by Andrews in 1869 [7]. He found that a fluid (carbon dioxide in his case) becomes very milky, i.e. opalescent, close to its critical point. Figure 3 illustrates this so-called critical opalescence on the example of the mixing critical point of hexane and methanol. At high temperatures, hexane and methanol are miscible and form a homogeneous clear fluid. The mixture phase-separates into a methanol-rich phase (below the meniscus) and a hexanerich phase (above the meniscus) at low temperatures. Close to the transition between the miscible and phase-separated regimes, the mixture becomes very milky.

What is causing the critical opalescence? The fact that the fluid becomes milky means that it strongly scatters visible light. This implies that it contains structures of a size comparable to the wavelength of light (about $1 \mu \mathrm{m}$ ), much larger than typical microscopic lengths such as the distance between molecules (a few $\AA$ ). Critical opalescence thus demonstrates that a critical point features strong fluctuations on large length scales.

Formally, these fluctuations can be characterized in terms of correlation functions. For the mixing critical point discussed above, one could define a concentration correlation function $G\left(\mathbf{x}-\mathbf{x}^{\prime}\right)=\left\langle\Delta c(\mathbf{x}) \Delta c\left(\mathbf{x}^{\prime}\right)\right\rangle$. Here $\Delta c(\mathbf{x})$ is the deviation of the hexane concentration $c$ at position $\mathbf{x}$ from its average value; and $\langle\ldots\rangle$ denotes the thermodynamic average. The appropriate correlation function for a liquid-gas critical point would be the density-density correlation function $\left\langle\Delta \rho(\mathbf{x}) \Delta \rho\left(\mathbf{x}^{\prime}\right)\right\rangle$; and for a magnetic critical point, it would be the spin-spin correlation function $\left\langle\Delta S(\mathbf{x}) \Delta S\left(\mathbf{x}^{\prime}\right)\right\rangle$. Under normal conditions, these correlation functions decay exponentially to zero for large distances $\left|\mathbf{x}-\mathbf{x}^{\prime}\right| \rightarrow \infty$, $G\left(\mathbf{x}-\mathbf{x}^{\prime}\right) \sim \exp \left(-\left|\mathbf{x}-\mathbf{x}^{\prime}\right| / \xi\right)$ which defines the correlation length $\xi$. Upon approaching 
TABLE 1. Definitions of the commonly used critical exponents using the example of a ferromagnetic transition. $m$ is the magnetization and $h$ is an external magnetic field. $r$ denotes the distance from the critical point and $d$ is the space dimensionality.

\begin{tabular}{cccc}
\hline & exponent & definition & conditions \\
\hline specific heat & $\alpha$ & $c \propto|r|^{-\alpha}$ & $r \rightarrow 0, h=0$ \\
order parameter & $\beta$ & $m \propto(-r)^{\beta}$ & $r \rightarrow 0-, h=0$ \\
susceptibility & $\gamma$ & $\chi \propto|r|^{-\gamma}$ & $r \rightarrow 0, h=0$ \\
critical isotherm & $\delta$ & $h \propto|m|^{\delta} \operatorname{sign}(m)$ & $h \rightarrow 0, r=0$ \\
\hline correlation length & $v$ & $\xi \propto|r|^{-v}$ & $r \rightarrow 0, h=0$ \\
correlation function & $\eta$ & $G(\mathbf{x}) \propto|\mathbf{x}|^{-d+2-\eta}$ & $r=0, h=0$ \\
\hline dynamical & $z$ & $\xi_{\tau} \propto \xi^{z}$ & $r \rightarrow 0, h=0$ \\
activated dynamical & $\psi$ & $\ln \xi_{\tau} \propto \xi^{\psi}$ & $r \rightarrow 0, h=0$ \\
\hline
\end{tabular}

the critical point, the correlation length diverges as

$$
\xi \sim|r|^{-v}=\left|\left(T-T_{c}\right) / T_{c}\right|^{-v}
$$

where the reduced temperature $r=\left(T-T_{c}\right) / T_{c}$ is a dimensionless measure of the distance from criticality and $v$ is the correlation length critical exponent.

Fluctuations close to criticality are not only large but also slow. One can define a time scale, the correlation time $\xi_{\tau}$ in analogy to the correlation length $\xi$. The correlation time also diverges at the critical point,

$$
\xi_{\tau} \sim \xi^{z} \sim r^{-v z}
$$

which defines the dynamical critical exponent $z$. The fact that the fluctuations become arbitrarily slow is also called the critical slowing down. It does occur not just in real experimental systems, it also greatly hampers the efficiency of computer simulations of critical phenomena. ${ }^{4}$

Power-law singularities in the length and time scales at a critical point generically lead to power-law singularities in observable quantities. For example, at a ferromagnetic critical point, the magnetic susceptibility diverges as $\chi \sim\left|T-T_{c}\right|^{-\gamma}$, and the magnetization vanishes as $m \sim\left|T-T_{c}\right|^{\beta}$. Here, $\beta$ is the order parameter critical exponent, and $\gamma$ is called the susceptibility exponent. Analogously, at a liquid-gas critical point, the compressibility diverges as $\kappa \sim\left|T-T_{c}\right|^{-\gamma}$, and the density difference between the liquid and gas phases vanishes as $\Delta \rho \sim\left|T-T_{c}\right|^{\beta}$. Other observables show similar power laws. A summary of the commonly used critical exponents is given in Table 1, using the example of a ferromagnetic transition. The collection of all these power laws characterizes the critical point and is usually called the critical behavior.

\footnotetext{
4 In some cases, critical slowing down in computer simulations can be circumvented by clever "cluster" algorithms $[9,10]$, in other cases it is unavoidable.
} 
The values of the critical exponents generally do not depend on the microscopic details but only on the space dimensionality and the symmetries of the system under consideration. For instance, all liquid-gas critical points have the same exponents. This phenomenon, called universality, is very fortunate for theory, because it permits the determination of the exact critical exponent values from simplified model problems as long as they have the correct symmetries and dimensionalities. It also permits the definition of universality classes comprising all critical points that share the same critical exponents.

Above, we have seen that fluctuations become very strong as one approaches a critical point. Do they influence the critical behavior? The answer to this question depends on the space dimensionality $d$. In general, fluctuations become less important with increasing dimensionality. ${ }^{5}$ In sufficiently low dimensions, fluctuations are so strong that they completely destroy the ordered phase at all (nonzero) temperatures. Consequently, there is no phase transition. This happens for $d \leq d_{c}^{-}$where $d_{c}^{-}$is the so-called lower critical dimension. Between $d_{c}^{-}$and the upper critical dimension $d_{c}^{+}$, an ordered phase and thus a phase transition exist, but the critical exponents are influenced by fluctuations and depend on $d$. Finally, for $d>d_{c}^{+}$, fluctuations are unimportant for the critical behavior. The exponents become independent of $d$ and take their mean-field values. For example, for Ising ferromagnets, the critical dimensions are $d_{c}^{-}=1$ and $d_{c}^{+}=4$, for Heisenberg ferromagnets they are $d_{c}^{-}=2$ and $d_{c}^{+}=4$.

\section{Scaling}

Scaling theory is a phenomenological description of critical points. It is an extremely powerful tool for the analysis of experimental or numerical data. Scaling theory was first put forward by Widom [11] as the scaling hypothesis. Within modern renormalization group theory [12], it can be derived from first principles.

The basic idea of scaling theory is that the correlation length $\xi$ is the only relevant length scale close to criticality. Right at the critical point, $\xi$ diverges, and the system is scale-invariant. Rescaling all lengths by a common factor should therefore leave the system unchanged. A little bit off criticality, $\xi$ is large but finite. Thus, if we rescale all lengths, we need to adjust the external parameters (temperature, field, etc.) such that $\xi$ has the same value. After that, the system should again be unchanged.

Let us now apply this idea to the free energy density $f=F / V$ of a system near a ferromagnetic critical point, written as function of the reduced temperature $r$ and the external magnetic field $h$. Rescaling all lengths by an arbitrary common factor $b$ leads to the homogeneity relation

$$
f(r, h)=b^{-d} f\left(r b^{1 / v}, h b^{y_{h}}\right)
$$

where the exponent $y_{h}$ is related to $\delta$ by $y_{h}=d \delta /(1+\delta)$. The factor $b^{-d}$ arises from the change of volume upon the rescaling, the changes in the arguments of $f$ reflect

\footnotetext{
${ }^{5}$ Intuitively, this happens because the number of neighbors of a given site increases with the dimensionality. A large number of neighbors effectively averages out the fluctuations felt by a particular site.
} 
the adjustments necessary to keep $\xi$ at its old value. Homogeneity relations for other thermodynamic variables can be obtained simply by taking the appropriate derivatives of (3). For example, the specific heat is given by a second derivative of the free energy, $c \sim-\partial^{2} f / \partial r^{2}$. Its scaling form thus reads

$$
c(r, h)=b^{-d+2 / v} c\left(r b^{1 / v}, h b^{y_{h}}\right) .
$$

How can homogeneity relations such as (3) and (4) be used to analyze experimental or numerical data? The key is that the homogeneity relations hold for arbitrary $b$, implying that we can set $b$ to whatever value we find useful. For example, if we were interested in the dependence of $c$ on the reduced temperature at zero field, we could set $b=r^{-v}$. This yields $c(r, 0)=r^{d v-2} c(1,0)$ which could be used to determine the exponent $\alpha=2-d v$ via a fit of the data.

The scaling form (3) of the free energy density $f$ depends on only two exponents. Since all thermodynamic observables can be determined from $f$, this implies that the various exponents listed in Table 1 are not independent. Instead they are related by the so-called scaling relations $2-\alpha=2 \beta+\gamma$ and $2-\alpha=\beta(\delta+1)$ as well as the hyperscaling relations $2-\alpha=d v$ and $\gamma=(2-\eta) v$.

We note that scaling in the form discussed here holds only below the upper critical dimension $d_{c}^{+}$. Above $d_{c}^{+}$, the situation is more complicated, and we refer the reader to a textbook about critical phenomena such as Ref. [4].

\section{Landau theory}

Landau $[13,14,15,16]$ provided a general framework for the description of phase transitions. According to Landau, bulk phases can be distinguished according to their symmetries, and phase transitions involve the (spontaneous) breaking of these symmetries. ${ }^{6}$ As an example, consider the phase transition in an easy-axis (Ising) ferromagnet, i.e., a ferromagnet in which the spins prefer to be either up or down (parallel or antiparallel to a particular crystallographic direction). In the paramagnetic phase at high temperatures, the spins point up or down at random, implying that the global up-down symmetry is not broken. In contrast, in the ferromagnetic low-temperature phase, the spins spontaneously pick a preferred direction (either up or down). Thus, the up-down symmetry is spontaneously broken.

A central concept in Landau's framework is the order parameter, a physical quantity that characterizes the degree of symmetry breaking. It is zero in one phase (the disordered phase) and nonzero and usually nonunique in the other phase (the ordered phase). In our example of an Ising ferromagnet, the total magnetization $m$ is an order parameter. $m$ vanishes in the paramagnetic phase while it is nonzero in the ferromagnetic phase. Moreover, $m$ is nonunique (positive or negative) as the magnetization can be either up or down spontaneously.

\footnotetext{
6 In recent years, there has been an increasing interest in phase transitions that do not follow Landau's paradigm. These unconventional transitions often involve topological degrees of freedom (see, e.g., Refs. $[17,18]$ for introductory discussions). They are beyond the scope these lectures.
} 


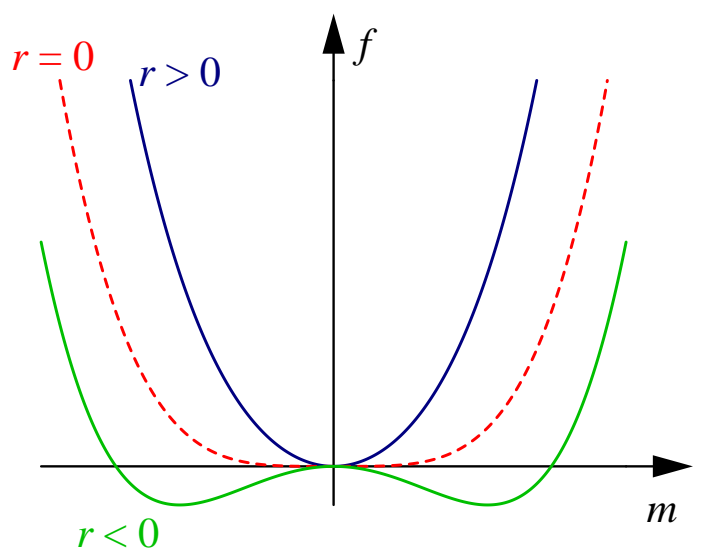

FIGURE 4. Sketch of the Landau free energy density $f$ as function of the order parameter $m$.

In addition to providing the general framework for phase transitions discussed above, Landau also developed an (approximate) quantitative description which is now known as the Landau theory of phase transitions. It is based on the idea that the free energy density $f$ close to the transition can be expanded in powers of the order parameter $m$,

$$
f=-h m+r m^{2}+v m^{3}+u m^{4}+\ldots .
$$

In the case of the ferromagnetic transition, the first term represents the external magnetic field. Moreover, the cubic coefficient $v$ and all higher-order odd terms must vanish because the free energy must be up-down symmetric in the absence of a field. For other phase transitions (such as the nematic transition in liquid crystals), odd terms do occur in the Landau expansion of the free energy. The coefficients $r, v$, and $u$ depend on the material as well as the external conditions (temperature, pressure, etc.). The correct physical state for given values of these coefficients is given by the minimum of $f$ with respect to the order parameter $m$.

The qualitative behavior of Landau theory can be easily discussed by plotting the free energy density $f(m)$. Let us assume a vanishing external field, $h=0$, as well as $v=0$ and $u>0 .^{7}$ Figure 4 shows $f(m)$ for several values of the quadratic coefficient $r$. For $r>0$, the free energy has a single minimum at $m=0$. Thus, $r>0$ corresponds to the paramagnetic phase. Conversely, the free energy has two degenerate minima at nonzero $m$ for $r<0$, separated by a local maximum at $m=0$. This means, the system is in the ferromagnetic phase for $r<0$. The transition between these two phases occurs at $r=0$ implying that $r$ is indeed a measure of the distance from the transition, $r \sim T-T_{c}$.

Quantitative results follow from solving $(\partial f / \partial m)=0$. In zero external field, $h=0$, the magnetization behaves as $m \sim(-r)^{1 / 2}$ for $r<0$ while the magnetic susceptibility diverges as $\chi=(\partial m / \partial h) \sim|r|^{-1}$. Thus, Landau theory predicts a continuous phase

${ }^{7}$ If $u$ were negative, the theory could not be truncated after the quartic term because it would be unstable against diverging order parameter $m$. 
transition, i.e., a critical point. ${ }^{8}$ On the critical isotherm, $r=0$, the magnetization varies as $m \sim h^{1 / 3}$ with the external field. In summary, Landau theory gives mean-field values for the critical exponents, viz., $\beta=1 / 2, \gamma=1$, and $\delta=3$.

How good is Landau theory? Landau theory does not contain any fluctuations because the order parameter is treated as a constant. Landau theory therefore gives the correct critical behavior if the dimensionality is above the upper critical dimension $d_{c}^{+}$of the problem. In contrast, Landau theory fails below $d_{c}^{+}$. In order to find the critical behavior for $d<d_{c}^{+}$, one needs to generalize Landau theory by making the order parameter a fluctuating field $m(\mathbf{x})$ that depends on position. The Landau free energy gets replaced by a functional, the so-called Landau-Ginzburg-Wilson (LGW) functional

$$
F[m(\mathbf{x})]=\int d^{d} x\left[-h m(\mathbf{x})+r m^{2}(\mathbf{x})+(\nabla m(\mathbf{x}))^{2}+u m^{4}(\mathbf{x})+\ldots\right] .
$$

The gradient term punishes rapid changes of the magnetization, it thus encodes the ferromagnetic interaction between neighboring spins. The partition function is now given by a functional integral

$$
Z=\int D[m(\mathbf{x})] \exp (-F[m(\mathbf{x})])
$$

In contrast to Landau theory, the problem defined by equations (6) and (7) cannot be solved by elementary means. However, it can be attacked successfully using modern renormalization group techniques [12].

\subsection{Introduction to quantum phase transitions}

\section{What is a quantum phase transition?}

The phase transitions we have considered so far all occur at nonzero temperatures, and they are often tuned by changing the temperature. At these transitions, the ordered phase is destroyed by thermal fluctuations. For instance, ice melts because the thermal motion of the water molecules destroys the crystal lattice; and a ferromagnetic material becomes paramagnetic at the Curie point due to the thermal motion of the spins.

A different kind of phase transition occurs at the absolute zero of temperature (in the quantum ground state) when a (nonthermal) parameter such as pressure, magnetic field, or chemical composition is varied. Consider, for example, the magnetic phase diagram of $\mathrm{LiHoF}_{4}$ shown in Fig. 5. $\mathrm{LiHoF}_{4}$ is an easy-axis (Ising) ferromagnet. In the absence of an external field, the material is in a ferromagnetic phase for temperatures below the Curie point of about $1.5 \mathrm{~K}$. If the temperature is raised above this value, the thermal motion of the holmium spins (which carry the magnetism) destroys ferromagnetic longrange order. This phase transition is a thermal transition of the type discussed in the last

\footnotetext{
${ }^{8}$ If the cubic coefficient in the free energy (5) does not vanish, or if we have a free energy with a negative quartic coefficient $u$ but a positive 6th-order coefficient, the theory yields a first-order transition.
} 


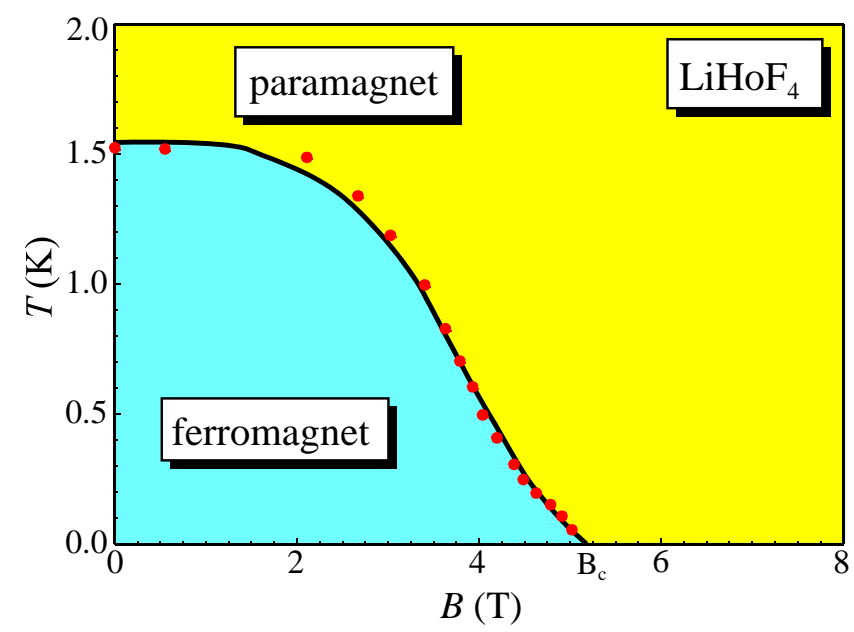

FIGURE 5. Magnetic phase diagram of $\mathrm{LiHoF}_{4}$ as function of temperature $T$ and transverse magnetic field $B$. The red dots represent experimental data from Ref. [19].

section. However, the figure also shows another way to destroy the ferromagnetism: If one applies a magnetic field $B$ perpendicular (transverse) to the preferred direction of the spins, the ferromagnetic order is suppressed. Beyond a critical field of about $5 \mathrm{~T}$, the ferromagnetic phase vanishes completely, even at zero temperature. Thus $\mathrm{LiHoF}_{4}$ undergoes a zero-temperature ferromagnetic phase transition at this critical magnetic field.

How does the magnetic field destroy the ferromagnetic phase? A qualitative understanding can be gained from considering a toy Hamiltonian, the transverse-field Ising model. ${ }^{9}$ It reads

$$
H=-J \sum_{\langle i j\rangle} \sigma_{i}^{z} \sigma_{j}^{z}-B \sum_{i} \sigma_{i}^{x}
$$

where the Pauli matrices $\sigma_{i}^{z}$ and $\sigma_{i}^{x}$ represent the $z$ and $x$ components of the spin operator at site $i$. The first term implements an attractive interaction between nearest neighbor sites of a cubic lattice. It prefers ferromagnetic order in the $z$-direction. The second term represents the transverse magnetic field (in the $x$-direction). To understand its effect, remember that $\sigma_{i}^{x}$ can be decomposed into spin-flip operators, $\sigma_{i}^{x}=\sigma_{i}^{+}+\sigma_{i}^{-}$. Thus, the transverse field term induces spin flips from up to down and from down to up. If the field becomes sufficiently strong, these spin flips destroy the ferromagnetic long-range order, even at zero temperature.

The zero-temperature phase transition between a ferromagnetic ground state for weak field $B$ and a paramagnetic ground state for strong $B$ is therefore driven by quantum fluctuations, i.e., quantum zero-point motion, rather than thermal fluctuations. It can be viewed as a consequence of Heisenberg's uncertainty principle. For this reason, this type

\footnotetext{
9 Note that the microscopic physics of $\mathrm{LiHoF}_{4}$ is significantly more complicated than the transverse-field Ising model. In particular, the Ho spins are not $S=1 / 2$ spins, their interaction is of dipolar type, and the hyperfine coupling between the electronic and nuclear magnetic moments is anomalously large [19].
} 

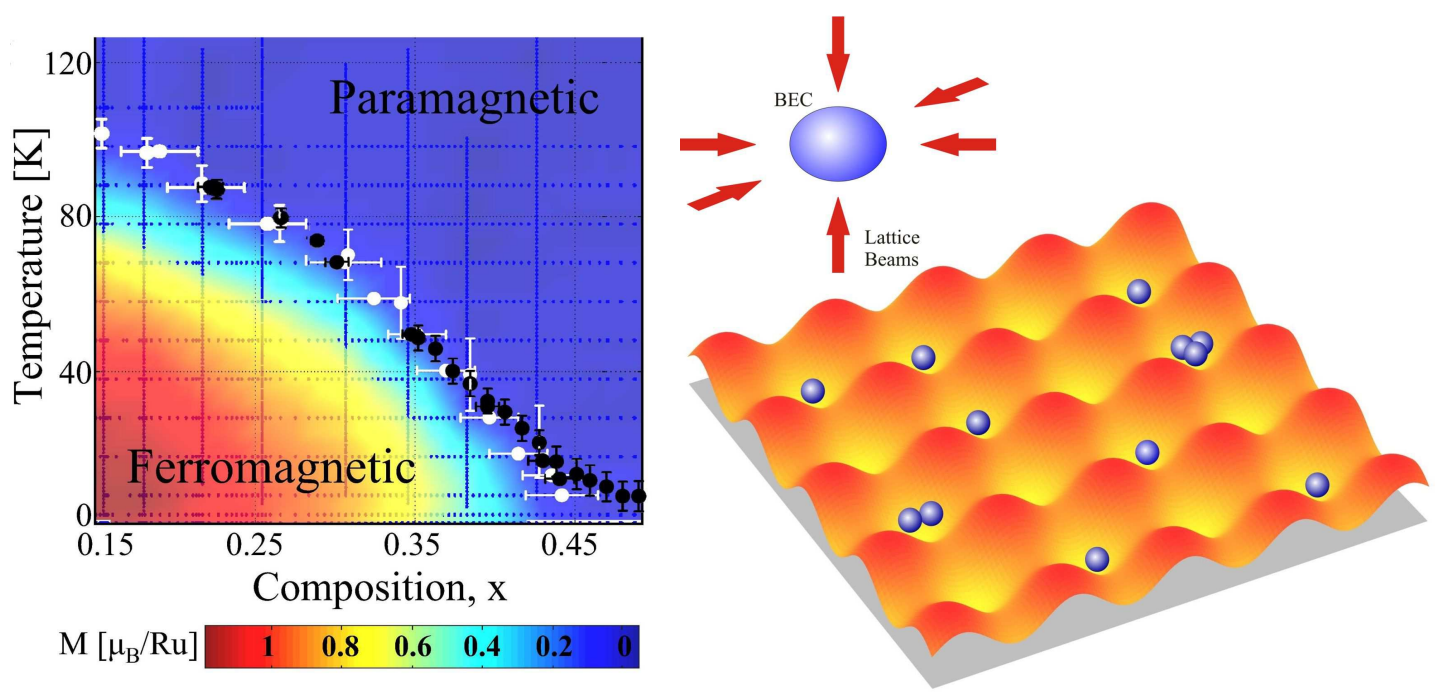

FIGURE 6. Examples of quantum phase transitions. Left: Magnetic phase diagram of the alloy $\mathrm{Sr}_{1-x} \mathrm{Ca}_{x} \mathrm{RuO}_{3}$. This material undergoes a quantum phase transition from a ferromagnetic metal to a paramagnetic metal as function of the Ca concentration $x$ (after Ref. [20]). Right: Mott superfluid-insulator transition of an ultracold gas of bosons in an optical lattice produced by standing laser waves. If the optical lattice is weak, the ground state is a coherent superfluid. For strong lattice potential, the atoms localize in the wells, resulting in an insulating ground state (see Ref. [21] for an experimental realization of this idea).

of phase transition is called a quantum phase transition. Quantum phase transitions can be divided into first-order and continuous just like thermal phase transitions. First order quantum phase transitions correspond to simple energy level crossings at which two different ground states are exactly degenerate. Continuous quantum phase transitions, i.e., quantum critical points, involve diverging length and time scales of the quantum fluctuations.

Quantum phase transitions have been identified in a wide variety of condensed matter systems. Examples include ferromagnetic and antiferromagnetic transitions in strongly correlated electron materials, metal-insulator transitions in doped semiconductors and metals, as well as superfluid-insulator transitions in ultracold atomic gases (see Fig. 6).

\section{Quantum-to-classical mapping}

Can we generalize the concepts for the description of phase transitions introduced in the last section (such scaling and Landau theory) to the case of quantum phase transitions? An important idea that helps addressing this question is the so-called quantumto-classical mapping.

Consider the partition function of a classical many-particle system. It can be written as a (high-dimensional) phase space integral. If the Hamiltonian is the sum of a kinetic part the depends only on momenta and a potential part that depends only on positions, $H(\mathbf{p}, \mathbf{q})=T(\mathbf{p})+V(\mathbf{q})$ (as is often the case), the partition function factorizes according 
to

$$
Z=\int d \mathbf{p} d \mathbf{q} \exp [-\beta H(\mathbf{p}, \mathbf{q})]=\int d \mathbf{p} \exp [-\beta T(\mathbf{p})] \int d \mathbf{q} \exp [-\beta V(\mathbf{q})]
$$

where $\beta=1 /\left(k_{B} T\right)$ is the inverse temperature. The kinetic part is (usually) a product of independent Gaussian integrals; it can not produce any singularities. The phase transition physics must therefore originate in the configuration part of the partition function. Consequently, only fluctuations in space but not in time need to be considered in the description of a classical phase transition. ${ }^{10}$ This explains why models without any internal dynamics such as the classical Ising model or our classical LGW theory (6) correctly describe the thermodynamics of classical phase transitions.

In a quantum system, a factorization of the partition function into kinetic and potential parts analogous to (9) is generally impossible because the operators of the kinetic energy, $T(p)$, and the potential energy, $V(q)$, do not commute $\left(e^{A+B} \neq e^{A} e^{B}\right.$ if $A$ and $B$ do not commute). Thus, statics and dynamics are coupled, and we need to treat them on equal footing. The problem can be addressed by using the Trotter identity [22] $e^{A+B}=\lim _{N \rightarrow \infty}\left[e^{A / N} e^{B / N}\right]^{N}$, leading to

$$
Z=\operatorname{Tr} \exp [-\beta H]=\operatorname{Tr} \exp [-\beta(T+V)]=\lim _{N \rightarrow \infty} \prod_{n=1}^{N}(\exp [-\beta T / N] \exp [-\beta V / N]) .
$$

The Trotter decomposition effectively cuts the inverse temperature $\beta$ into many "slices". As $\beta$ plays the role of an imaginary time, ${ }^{11}$ this introduces an extra coordinate $\tau$, the imaginary time coordinate that goes from 0 to $\beta$ in steps of $\beta / N$. At zero temperature, this direction becomes infinitely long. We thus conclude that imaginary time plays the role of an additional dimension at a quantum phase transition. By inserting appropriate resolutions of the unit operator between the factors on the r.h.s. of (10), one can rewrite the partition function as a functional integral ${ }^{12}$

$$
Z=\int D[q(\tau)] \exp \{-S[q(\tau)]\}
$$

As a result, we arrive at the famous quantum-to-classical mapping which states: $A$ quantum phase transition in $d$ space dimensions is equivalent to a classical (thermal) phase transition in $d+1$ dimensions.

This mapping is a very powerful tool that we will use repeatedly to understand the properties of new quantum phase transitions. However, it comes with a number of caveats. (i) The mapping works for thermodynamic quantities only, as it is based on an analysis of the partition function. It is not applicable to other properties of quantum phase transitions such as real-time dynamics and transport properties. (ii) The classical $(d+1)$-dimensional system arising from the mapping can be unusual and anisotropic. (iii) The mapping only works if the resulting action $S[q(\tau)]$ is real such that it can be

\footnotetext{
10 This argument holds for the thermodynamics which is governed by the partition function but not for the real-time dynamics at a classical transition.

11 The Boltzmann factor $\exp (-\beta H)$ looks like a time evolution operator $\exp (-i t H / \hbar)$ with imaginary $t$.

12 This is analogous to deriving Feynman's path integral for the propagator in quantum mechanics [23].
} 
TABLE 2. Analogies between important quantities in the quantum-to-classical mapping (after Ref. [24]).

\begin{tabular}{cc}
\hline Quantum System & Classical System \\
\hline$d$ space, 1 time dimensions & $d+1$ space dimensions \\
quantum coupling constant & classical temperature $T$ \\
inverse physical temperature $1 /\left(k_{B} T\right)$ & finite size in the imaginary time direction \\
spatial correlation length $\xi$ & spatial correlation length $\xi$ \\
inverse energy gap $1 / \Delta$ & correlation length $\xi_{\tau}$ in the imaginary time direction \\
\hline
\end{tabular}

interpreted as a classical free energy functional. As we will see later, this is the case for some quantum phase transitions while others lead to complex actions, for example due to Berry phases. ${ }^{13}$ In some cases, it actually depends on the details of the mapping procedure [for instance, on which sets of basis states are used in the decomposition of (10)] whether or not the resulting action is real. A summary of the relations between the quantum system and its classical counterpart is given in Table 2.

\section{Scaling at a quantum critical point}

With the quantum-to-classical mapping in mind, we can generalize Widom's scaling hypothesis to quantum phase transitions. To do so, we need to include the imaginary time variable $\tau$ in the scale transformation. In general, space and imaginary time do not scale in the same way. Thus, if we scale lengths according to $L \rightarrow b L$, we need to scale imaginary time by a different factor, i.e., $\tau \rightarrow b^{z} \tau$. (Remember, the dynamical exponent $z$ relates the divergencies of length and time scales at a critical point.)

Moreover, even though a quantum phase transition occurs at exactly zero temperature, experiments are performed at nonzero temperatures. It is therefore useful to include the temperature as an independent external parameter from the outset. ${ }^{14}$ As the temperature is an energy, it scales like an inverse time, i.e., $T \rightarrow b^{-z} T$. Consequently, the scaling form of the free energy density at a quantum critical point reads

$$
f(r, h, T)=b^{-(d+z)} f\left(r b^{1 / v}, h b^{y_{h}}, T b^{z}\right) .
$$

Note that $r$ measures the distance from the quantum critical point along the quantum tuning parameter axis (for example the transverse magnetic field axis in the case of the transverse-field Ising model), it is not related to the temperature. Scaling forms of thermodynamic observables can again be obtained by taking the appropriate derivatives; and those of other quantities (such as correlation functions) can be constructed analogously.

\footnotetext{
13 If the action $S[q(\tau)]$ is complex, the corresponding Boltzmann factor $\exp \{[-S[q(\tau)]\}$, which acts as statistical weight in the path integral (11), is not positive definite. This is known as the notorious sign problem; it strongly hampers quantum Monte-Carlo simulations of such systems.

14 Under the quantum-to-classical mapping, the inverse temperature corresponds to the system size in imaginary time direction. Temperature scaling at a quantum critical point is thus equivalent to finite size scaling [25] in the corresponding (mapped) classical system.
} 
Along the same lines, one can also generalize the Landau-Ginzburg-Wilson (LGW) order parameter field theory (6) to quantum phase transitions. Since we need to include fluctuations in space and (imaginary) time, the order parameter $m$ becomes a function of $\mathbf{x}$ and $\tau$. Expanding in powers of $m$ as well as in gradients and time derivatives gives the LGW action or free energy

$$
S[m(\mathbf{x}, \tau)]=\int d^{d} x d \tau\left[-h m+r m^{2}+(\nabla m)^{2}+\left(1 / c^{2}\right)(\partial m / \partial \tau)^{2}+u m^{4}+\ldots\right] .
$$

Here, $c$ plays the role of a propagation speed for the order parameter fluctuations. It must be emphasized that (13) is just the simplest example of a quantum LGW theory. It applies. e.g., to the ferromagnetic transition in the transverse-field Ising model. Many other quantum phase transitions have more complicated LGW theories. In particular, if the system contains soft (gapless) excitations other than the order parameter fluctuations, the expansion in powers of time derivatives generally breaks down. The resulting time/frequency dependence then becomes nonanalytic [26, 27, 28]. We will see examples of this behavior in later sections (when we discuss the effects of dissipation).

\section{Phase diagram close to a quantum critical point}

The phase diagram of a system close to a quantum critical point is very rich; it contains several qualitatively different regions depending on the presence or absence of longrange order as well as the character of the fluctuations. In particular, it is important to distinguish thermal and quantum fluctuations. A fluctuation is of (predominantly) thermal character if its frequency $\omega_{c}$ is below the thermal energy, $\hbar \omega_{c} \ll k_{B} T$. In the opposite case, $\hbar \omega_{c} \gg k_{B} T$, the fluctuation is of quantum character.

Figure 7 shows a schematic of such a phase diagram. The disordered phase consists of three different regions. In the thermally disordered region, located on the ordered side of the quantum critical point but at high temperatures, the long-range order is destroyed by thermal fluctuations. In the quantum disordered region located at the disordered side of the quantum critical point at low temperatures, quantum fluctuations destroy the longrange order while thermal effects are unimportant (even the ground state is disordered). Between these regions is the so-called quantum critical region, located at $B \approx B_{c}$ and, somewhat counter-intuitively, at comparatively high temperatures where the character of the fluctuations is thermal. In this regime, the system is critical with respect to $B$, and the critical singularities are cut-off exclusively by the temperature.

It is important to note that the asymptotic critical behavior at any nonzero temperature is dominated by thermal fluctuations because the characteristic frequency $\omega_{c} \sim \xi_{\tau}^{-1}$ vanishes due to critical slowing down. However, the classical critical region around the phase boundary becomes very narrow at low temperatures. Another way to understand this result is to compare the correlation time $\xi_{\tau}$ with the system size $\beta=1 /\left(k_{B} T\right)$ in imaginary time direction. Upon approaching the phase boundary, $\xi_{\tau}$ diverges. A any nonzero temperature, it thus eventually becomes larger than the system size $\beta$. At this point, the imaginary time dimension drops out, and the critical behavior becomes classical. 


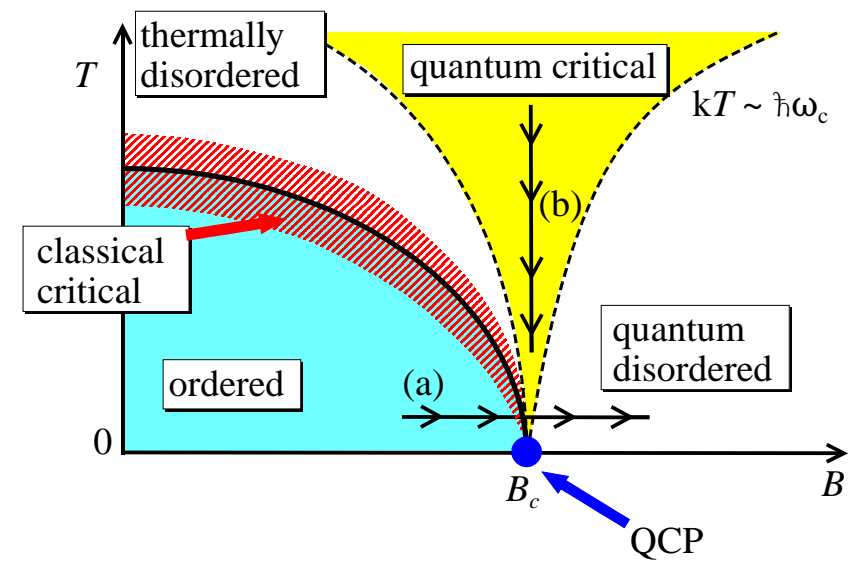

FIGURE 7. Schematic phase diagram in the vicinity of a quantum critical point (QCP). The thick solid line marks the boundary between the ordered and disordered phases. The dashed lines indicate the crossover between predominantly quantum or classical character of the fluctuations. In the hatched classical critical region, quantum mechanics is unimportant, and the leading classical critical singularities can be observed. Experiments following path (a) and (b) are discussed in the text.

We thus conclude that the asymptotic critical behavior is quantum only at precisely zero temperature while it is classical for any nonzero temperature. This justifies calling all finite-temperature phase transitions classical even if the underlying microscopic physics is quantum. Nonetheless, the quantum critical point controls large portions of the phase diagram at nonzero temperatures. An experiment along path (a) in figure 7 will see the classical behavior only in an extremely narrow range around the transition. Moreover, an experiment along path (b) in the quantum critical region explores the temperature scaling of the quantum critical point.

\section{PHASE TRANSITIONS IN DISORDERED SYSTEMS}

In this section, we consider various types of disorder or randomness and derive criteria that govern under what conditions they can influence a phase transition.

\subsection{Types of disorder}

\section{Quenched vs. annealed disorder}

Disorder ${ }^{15}$ or randomness in a physical system can have many different origins including vacancies or impurity atoms in a crystal as well as extended defects of the crystal

15 Unfortunately, the word "disorder" is used in two different meanings in the field of phase transitions. First, it is used to characterize a phase without long-range order in the Landau sense. In the case of the ferromagnetic transition, for example, we call the paramagnetic phase a disordered phase. Second, the word "disorder" denotes imperfections or randomness of the underlying system. This is the meaning here. 
lattice such as dislocations or grain boundaries. The solid could also be amorphous rather than crystalline. In ultracold atom systems, disorder can be realized, for example, using speckle laser light.

To understand the physical consequences of such disorder, it is important to distinguish quenched and annealed disorder. Quenched or frozen-in disorder is static; this means that the impurities or defects do not move or change over the relevant experimental time scales. In contrast, annealed disorder fluctuates during the duration of the experiment. From a conceptually point of view, annealed disorder is easier to deal with. If the disorder degrees of freedom (for example, the impurity positions) are in thermal equilibrium over the experimental time scales, they can simply be included in the usual statistical mechanics description of the system. In other words, the thermodynamics of a system with annealed disorder is obtained by averaging the partition function over the impurity degrees of freedom, $Z_{a v}=[Z]_{d i s}$ where $[\ldots]_{d i s}$ denotes the disorder average.

In contrast, every sample, i.e., every disorder realization, is different in the case of quenched disorder. To obtain average thermodynamic quantities one thus needs to average the free energy or, equivalently, the logarithm of the partition function, $\ln Z_{a v}=$ $[\ln Z]_{\text {dis. }}{ }^{16}$ Technically, averaging $\ln Z$ is much harder than averaging $Z$ itself; and this makes the physics of quenched disordered systems a difficult research area. One way to overcome this difficulty is the so-called replica trick [29]. It involves writing $\ln Z=$ $\lim _{n \rightarrow 0}\left(Z^{n}-1\right) / n$ and averaging $Z^{n}$ before taking the limit $n \rightarrow 0$. This method has met some success, for example, in the theory of spin glasses. However, exchanging the average and the $n \rightarrow 0$ limit is mathematically problematic; and the replica approach is known to fail in some cases. We will not be using this method.

In the remainder of these lectures, we will not consider annealed disorder but focus on the more interesting (and complicated) case of quenched disorder.

\section{Random mass, random fields, and all that}

In Section 1, we have seen that the qualitative properties of phase transitions depend on the space dimensionality and on symmetries but not on microscopic details. This suggests that we should classify the various kinds of quenched disorder according to their symmetries.

Consider, for example, a clean (three-dimensional) classical ferromagnet described an Ising Hamiltonian

$$
H=-J \sum_{\langle i j\rangle} S_{i} S_{j}
$$

with spins $S_{i}= \pm 1$. The sum goes over pairs of nearest neighbors on a cubic lattice, and $J>0$ is the exchange interaction. How could disorder change this Hamiltonian? One possibility is that the exchange interaction becomes nonuniform and varies randomly from place to place. (In a real system this could be due to nonmagnetic impurity atoms

\footnotetext{
16 A complete description of a large ensemble of samples strictly requires working out the probability distributions of observables rather than just the average. This will become an important point later in this article.
} 
modulating the distances between the spins.) The disordered Hamiltonian reads

$$
H=-\sum_{\langle i j\rangle} J_{i j} S_{i} S_{j}
$$

where the $J_{i j}$ are random variables. As long as all $J_{i j}$ remain positive, such randomness is a weak and rather benign type of disorder. It does not change the two bulk phases: At sufficiently low temperatures, the system is still a ferromagnet, and at sufficiently high temperatures it is still a paramagnet. Moreover the up-down spin symmetry is not broken by the disorder. The disorder just changes the local tendency towards ferromagnetism, in other words it changes the "local critical temperature." Consequently, this type of disorder is often called random- $T_{c}$ disorder. In a LGW description of the transition, such disorder would appear as a random variation $\delta r(\mathbf{x})$ of the distance from criticality,

$$
F[m(\mathbf{x})]=\int d^{d} x\left\{-h m(\mathbf{x})+[r+\delta r(\mathbf{x})] m^{2}(\mathbf{x})+(\nabla m(\mathbf{x}))^{2}+u m^{4}(\mathbf{x})+\ldots\right\} .
$$

The disorder couples to the $m^{2}$ term in the LGW free energy functional. In quantum field theory, this term is usually called the mass term. Therefore, random- $T_{c}$ disorder is also called random-mass disorder. (In addition to random exchange couplings, random-mass disorder can also be realized by random dilution of the spins.)

Alternatively, we could imagine disorder that couples linearly to the order parameter $m$ rather than $m^{2}$. In our example ferromagnet, this corresponds to a magnetic field that varies randomly from site to site. The LGW theory of such a system reads

$$
F[m(\mathbf{x})]=\int d^{d} x\left\{-h(\mathbf{x}) m(\mathbf{x})+r m^{2}(\mathbf{x})+(\nabla m(\mathbf{x}))^{2}+u m^{4}(\mathbf{x})+\ldots\right\}
$$

where $h(\mathbf{x})$ is the random variable. This type of disorder is called random-field disorder. It locally breaks the up-down spin symmetry. Whether or not the symmetry is broken globally depends on the probability distribution of the random fields. A particularly interesting situation arises if the distribution is even in $h$ such that the up-down symmetry is globally preserved in the statistical sense. As we will see later in this section, randomfield disorder is generally stronger than random-mass disorder.

Many other types of disorder are possible. For example, in a Heisenberg magnet where the spin variables are three-dimensional unit vectors, the disorder could break the rotational symmetry in spin space in a random fashion. This defines random-anisotropy disorder. In a superconductor or superfluid, the disorder could lead to random phases of the complex order parameter. If the interactions in a spin system pick up random signs, the system becomes frustrated which often results in spin-glass behavior [30].

In these lectures, we will mostly consider the cases of random-mass and random-field disorder. 


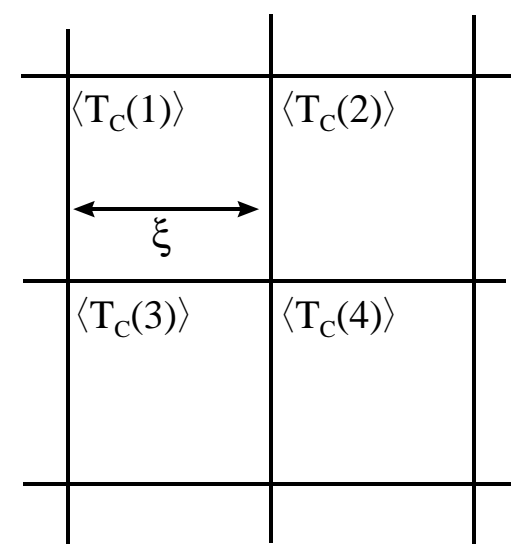

FIGURE 8. Derivation of the Harris criterion. The system is divided into blocks of linear size $\xi$. Due to the disorder, each block has its own local "critical temperature."

\section{Important questions}

If quenched disorder is added to a clean system undergoing a phase transition, a hierarchy of important questions arises naturally:

- Are the bulk phases (the phases separated by the transition) qualitatively changed by the disorder?

- Is the phase transition still sharp, or is it smeared because different parts of the system undergo the transition independently?

- If the phase transition is still sharp, does its order (first order vs. continuous) change?

- If the phase transition remains continuous, does the critical behavior, i.e., the values of the critical exponents, change?

\subsection{Random-mass disorder and the Harris criterion}

In this section, we consider a clean system undergoing a continuous phase transition (a critical point) and ask whether or not random-mass disorder changes the properties of the phase transition. (We already know from the discussion in the last section that the bulk phases will not change.)

Harris addressed this question in a famous paper [31] published in 1974. Specifically, he found a powerful criterion for the stability of a clean critical point against randommass disorder. To derive this criterion, imagine that we are at a temperature somewhat above the (global) critical temperature $T_{c}$ of the system. We can divide the system into blocks whose linear size is the correlation length $\xi$ (see Fig. 8). The spins within each block are effectively parallel and fluctuate together as a large superspin. Due to the disorder, each block $i$ has its own "local critical temperature" $T_{c}(i)$ which is determined 
by the values of the interactions in this block. ${ }^{17}$ Harris' idea consists in comparing the variations $\Delta T_{c}$ of the local critical temperature from block to block with the distance $T-T_{c}$ from the global phase transition point. If $\Delta T_{c}<T-T_{c}$, the blocks are all on the same side of the phase transition, and the system is more or less uniform. In contrast, for $\Delta T_{c}>T-T_{c}$, some blocks are in the disordered (paramagnetic) phase and some are in the ordered (ferromagnetic) phase, making a uniform transition impossible.

For the clean critical behavior to be stable we thus must have $\Delta T_{c}<T-T_{c}$ as we approach the transition, i.e., for $\xi \rightarrow \infty$. The dependence of $\Delta T_{c}$ on $\xi$ can be estimated using the central limit theorem. As the local $T_{c}(i)$ is determined by an average of a large number of random variables in the block [for example, the random $J_{i j}$ in the Hamiltonian (15)], its variations decay as the square root of the block volume,

$$
\Delta T_{c} \sim \xi^{-d / 2} .
$$

On the other hand, according to (1), the global distance from criticality is related to $\xi$ via

$$
T-T_{c} \sim \xi^{-1 / v}
$$

The condition $\Delta T_{c}<T-T_{c}$ for $\xi \rightarrow \infty$ therefore leads to the exponent inequality

$$
d v>2
$$

This is the famous Harris criterion for the stability of a clean critical point.

Let us discuss the interpretation of this inequality in more detail. If the Harris criterion $d v>2$ is fulfilled, the ratio $\Delta T_{c} /\left(T-T_{c}\right)$ goes to zero as the critical point is approached. This means, the system looks less and less disordered on larger length scales; and the effective disorder strength vanishes right at criticality. Consequently, the disordered system features the same critical behavior as the clean one. This also implies that all thermodynamic observables are self-averaging, i.e., their values are the same for all disorder realizations. An example of a transition that fulfills the Harris criterion is the ferromagnetic transition in a three-dimensional classical Heisenberg model. Its clean correlation length exponent is $v \approx 0.69>2 / d=2 / 3$.

In contrast, if $d v<2$, the ratio $\Delta T_{c} /\left(T-T_{c}\right)$ increases upon approaching the phase transition. This means, the blocks become effectively more different on larger length scales. Eventually, some blocks will be on one side of the transition while other blocks are on the other side. This makes a uniform sharp phase transition impossible. The character of the transition must therefore change, and the clean critical behavior is unstable. In the marginal case $d v=2$, more sophisticated methods are required to decide the stability of the clean critical point.

What is the fate of the transition if the Harris criterion is violated? The Harris criterion itself cannot answer this question, but research over the last four decades has established a number of possible scenarios. In the simplest case, the phase transition

\footnotetext{
17 It is important to realize that, generally, finite-size blocks cannot undergo a true phase transition at their respective local critical temperature $T_{c}(i)$. Instead, $T_{c}(i)$ marks the point where the spins in the block lock together to form the superspin.
} 
remains sharp and continuous but the critical behavior changes. The disordered system is in a new universality class featuring a correlation length exponent that fulfills the inequality $d v>2$. In this case, the disorder strength remains finite at large length scales, and observables at the critical point are not self-averaging. Many phase transitions in classical disordered systems follow this scenario, for example the three-dimensional classical Ising model. Its clean correlation length exponent is $v \approx 0.63$ which violates the Harris criterion. In the presence of random-mass disorder, the critical behavior therefore changes with the disordered correlation length exponent taking a value of about 0.68 .

Quantum phase transitions in disordered systems often display more exotic behavior (more dramatic changes than just different critical exponent values). We will explore several such phenomena in Sections 3 to 5.

Let us finish this section with a number of important remarks. (i) The Harris criterion holds in the same form, $d v>2$ for both classical (thermal) and quantum phase transitions. Specifically, this means that the dimensionality $d$ should not be replaced by $(d+1)$ or $(d+z)$ for a quantum phase transition (as one might have guessed from the quantumto-classical mapping). The reason is that the $d$ in the Harris criterion stems from averaging the disorder degrees of freedom using the central limit theorem. As quenched disorder is time-independent, the number of random variables in a block scales as $\xi^{d}$ for both classical and quantum phase transitions. This insight also implies that the Harris criterion needs to be modified for disorder that is perfectly correlated in one or more dimensions such as line or plane defects. In these cases, $d$ needs to be replaced by the number $d_{r}$ of dimensions in which there actually is randomness, i.e., $d_{r}=d-1$ for line defects and $d_{r}=d-2$ for plane defects. This greatly enhances the relevance of such perfectly correlated disorder.

(ii) The Harris criterion $d v>2$ applies to uncorrelated or short-range correlated disorder. If the disorder displays long-range correlations in space, the inequality needs to be modified because the central-limit theorem estimate of $\Delta T_{c}$ changes. Weinrib and Halperin [32] considered the case of power-law correlations that fall off as $\left|\mathbf{x}-\mathbf{x}^{\prime}\right|^{-a}$. They found that the Harris criterion does not change for $a>d$. If $a<d$, the inequality needs to replaced by $a v>2$. Thus, the criterion reads

$$
\min (d, a) v>2 .
$$

This implies that long-range correlated disorder is more dangerous for a clean critical point than short-range correlated disorder. In other words, a given clean critical point may be stable against uncorrelated disorder but unstable against disorder with sufficiently long-ranged correlations. ${ }^{18}$

(iii) The Harris inequality $d v>2$ involves the clean correlation length exponent. It does not put a bound on the correlation length exponent of the disordered system. However, Chayes et al. [33] showed (under some mild assumptions) that the correlation length exponent of a critical point in a disordered system has to fulfill the same inequality $d v \geq 2$. Counter examples to this results are sometimes reported in the literature. In is

\footnotetext{
18 At first glance, this result may appear counter-intuitive because long-range correlated disorder looks "less random". The crucial point is, however, that the long-range correlations favor the appearance of large fluctuations.
} 


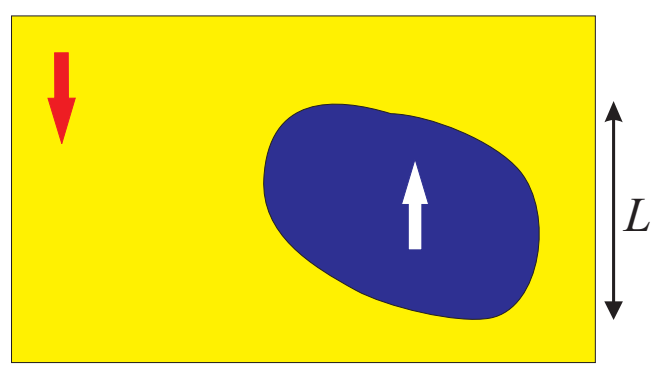

FIGURE 9. To derive the Imry-Ma criterion, one needs to compare the energy gain of a domain (of size $L$ ) due to aligning with the average random field with the energy loss due to the domain wall.

not always clear whether they are genuine violations of the theorem or whether they occur due to not fulfilling the underlying assumptions or maybe because the reported exponents are not in the true asymptotic critical region.

\subsection{Random-field disorder and the Imry-Ma argument}

In this section, we investigate a system undergoing a phase transition in the presence of random-field disorder. As an example, consider a (three-dimensional) Ising ferromagnet in a random magnetic field. Its Hamiltonian reads

$$
H=-J \sum_{\langle i j\rangle} S_{i} S_{j}-\sum_{i} h_{i} S_{i}
$$

Here, the $h_{i}$ are independent random variables with zero mean, $\left[h_{i}\right]_{d i s}=0$ and variance $\left[h_{i} h_{j}\right]_{d i s}=W \delta_{i j}$.

As discussed in Sec. 2.1, the random field locally breaks the up-down symmetry of the model. Spins on sites with positive random field, $h_{i}>0$, prefer pointing up $\left(S_{i}=1\right)$ while spins on sites with negative random field, $h_{i}<0$, prefer pointing down $\left(S_{i}=-1\right)$. This raises the question: Does a ferromagnetic phase (in which all spins align in the same direction) still exist in the presence of the random field? For strong random fields, this question can be easily answered in the negative. If $W \gg J^{2}$, almost all spins gain more energy from aligning with their local random fields rather than with each other. Thus, ferromagnetic order is impossible. What about weak random fields, $W \ll J^{2}$ ? In this case almost all spins will prefer to align with their neighbors rather than the field. One might be tempted to conclude that ferromagnetism survives in this case. However, this would be premature because long-range ferromagnetic order can be destroyed by the formation of domains.

Imry and Ma [34] developed a beautiful heuristic argument to test the stability of the ferromagnetic state against domain formation. Consider a single uniform spin-up domain of linear size $L$ (located in a region where the random field is mostly positive) embedded in a spin-down bulk system (see Fig. 9). According to Imry and Ma, we need to compare the energy gain due to aligning the domain with the average local random field with the energy loss due to the formation of the domain wall. In $d$ space dimensions, 


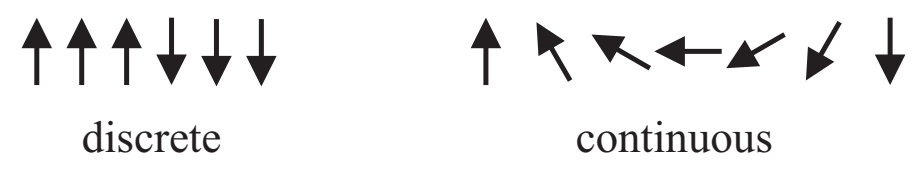

FIGURE 10. For discrete (Ising) symmetry, a domain wall is a "sharp" up-down spin flip. For continuous symmetry, the domain wall consists of a smooth change of the spin orientation over some distance.

the domain wall is a $(d-1)$-dimensional surface. Each bond crossing this surface has antiparallel spins and thus energy $+J$ rather than $-J$; it therefore contributes $2 J$ to the domain wall energy. As the number of such bonds is proportional to the domain wall area, we can estimate the domain wall energy to be

$$
\Delta E_{D W} \sim J L^{d-1}
$$

The energy gain due to aligning the uniform spin-up domain with the random field is simply given by the sum over all random field values in the domain, $\Delta E_{R F}=-\sum_{i}^{\prime} h_{i}$. As the $h_{i}$ are independent random variables, the typical value of this sum can again be estimated by means of the central limit theorem, giving

$$
\left|\Delta E_{R F}\right| \sim W^{1 / 2} L^{d / 2}
$$

Imry and Ma now observe that the uniform ferromagnetic state will be stable against the formation of domains if $\left|\Delta E_{R F}\right|<\Delta E_{D W}$ for all possible domain sizes $L$. Inserting the above estimates, this translates to $W^{1 / 2} L^{d / 2}<J L^{d-1}$. In the case of weak random field, $W \ll J^{2}$, this is certainly fulfilled for small domains containing just a few spins. Whether or not it also holds for large domains depends on the dimensionality $d$. If $d>2$, the domain wall energy increases faster with $L$ than the random field energy. Thus, domain formation is always unfavorable, and the ferromagnetic state is stable against weak random-field disorder.

In contrast, for $d<2$, the random field energy increases faster with $L$ than the domain wall energy. Even for weak random fields, there will be a critical $L$ beyond which forming domains that align with the local random field becomes favorable. Consequently, the uniform ferromagnetic state is unstable against domain formation for arbitrary random field strength. In other words, in dimensions $d<2$. random-field disorder prevents spontaneous symmetry breaking. Analyzing the marginal case, $d=2$, again requires more sophisticated methods.

Building on this heuristic argument, Aizenman and Wehr [35] proved rigorously that random fields destroy long-range order (and thus prevent spontaneous symmetry breaking) in all dimensions $d \leq 2$ for discrete (Ising) symmetry and in dimensions $d \leq 4$ for continuous (Heisenberg) symmetry. The difference between discrete and continuous symmetry can be easily understood by comparing the domain walls (see Fig. 10). In the discrete case, a domain wall consists of a sharp up-down spin flip at the surface of the domain. As estimated above, its energy is proportional to the surface area. i.e., it scales as $L^{d-1}$ with the domain size $L$. In the continuous case, the domain wall can be spread out over the entire domain, i.e., over a length of order $L$. The domain wall energy can be estimated within a LGW theory. The gradient term in (6) contributes 
$\Delta E_{D W} \sim L^{d}(\nabla m)^{2} \sim L^{d}(1 / L)^{2} \sim L^{d-2}$. The domain wall energy thus increases less rapidly with $L$ in the continuous case, making the system more susceptible towards domain formation. Inserting $\Delta E_{D W} \sim L^{d-2}$ into the Imry-Ma argument gives a critical dimensionality of 4 rather than 2 .

The above results hold for uncorrelated or short-range correlated random fields. Longrange correlated random fields with correlations that fall off as $\left|\mathbf{x}-\mathbf{x}^{\prime}\right|^{-a}$ modify the Imry-Ma argument because the energy gain due to aligning the domain with the local random field changes, provided $a<d$ [36]. In this case the uniform ferromagnetic state is stable for $a>2$ while domain formation is favored for $a<2$. In the opposite case, $a>d$, the uncorrelated Imry-Ma argument is unmodified.

As in the case of the Harris criterion, the Imry-Ma argument gives identical results for classical and quantum phase transitions because the dimensionality enters via the number of dimensions in which there is randomness. This means only space dimensions count but not the imaginary time dimension.

\subsection{Destruction of first-order phase transitions by disorder}

Reasoning very similar to the Imry-Ma argument can be used to attack a different problem, viz., the fate of a first-order phase transition in the presence of random-mass disorder.

Remember, a first order phase transition is characterized by macroscopic phase coexistence at the transition point. ${ }^{19}$ For example, at the liquid-gas phase transition of a fluid, a macroscopic liquid phase coexists with a macroscopic vapor phase. Random-mass disorder locally favors one phase over the other. We can thus pose the same question we asked in the last section: Will the macroscopic phases survive in the presence of the disorder or will the system form domains (droplets) that follow the local value of the random-mass term (the "local $T_{c}$ ")?

This question can be answered by adapting the Imry-Ma argument to the problem at hand $[37,38,35]$. Consider a single domain or droplet (of linear size $L$ ) of one phase embedded in the other phase. The free energy cost due to forming the surface is

$$
\Delta F_{\text {surf }} \sim \sigma L^{d-1}
$$

where $\sigma$ is the surface energy between the two phases. ${ }^{20}$ The energy gain from the random-mass disorder can be estimated via the central limit theorem, resulting in a typical magnitude of

$$
\left|\Delta F_{d i s}\right| \sim W^{1 / 2} L^{d / 2}
$$

where $W$ is the variance of the random-mass disorder.

\footnotetext{
19 Actually, the random-field problem considered in the last subsection can be viewed in the same way. Imagine adding an extra uniform field $h$ to the random-field Hamiltonian (22). As $h$ is tuned through zero at low temperatures, the system undergoes a first-order phase transition from the spin-down phase to the spin-up phase.

${ }^{20}$ The surface term scales as $L^{d-1}$ rather than $L^{d-2}$ because the two phases are generally not connected by a continuous transformation.
} 
The macroscopic phases are stable if $\left|\Delta F_{\text {dis }}\right|<\Delta F_{\text {surf }}$. Using the same reasoning as in the last subsection, this means that macroscopic phase coexistence is impossible in dimensions $d \leq 2$ no matter how weak the disorder is. In dimensions $d>2$, phase coexistence is possible for weak disorder but will be destabilized for sufficiently strong disorder.

We thus conclude that random-mass disorder destroys first-order phase transitions in dimensions $d \leq 2$. As before, this result holds for both classical (thermal) and quantum phase transitions [39] as long as the disorder is uncorrelated or short-range correlated in space. Long-range interactions can be taken into account as in the random-field case in the last section.

What is the ultimate fate of the phase transition if the first-order character is destroyed by the disorder? The Imry-Ma argument cannot answer this question. In many examples, the first-order transition is replaced by ("rounded to") a continuous one, but more complicated scenarios cannot be excluded.

\section{STRONG-DISORDER RENORMALIZATION GROUP}

After the introductory sections 1 and 2, we now turn to the main topic of this article, quantum phase transitions in disordered systems, taking the transverse-field Ising model as a paradigmatic example. We introduce a very powerful technique to attack this problem, the strong-disorder renormalization group (SDRG) which has become a standard tool in this field.

\subsection{Random transverse-field Ising chain}

We have already encountered the transverse-field Ising Hamiltonian (8) in Sec. 1.2 as a toy model for the quantum phase transition in $\mathrm{LiHoF}_{4}$. Here, we will be considering a one-dimensional disordered version of this model, the random transverse-field Ising chain. It is given by

$$
H=-\sum_{i} J_{i} \sigma_{i}^{z} \sigma_{i+1}^{z}-\sum_{i} B_{i} \sigma_{i}^{x}
$$

The interactions $J_{i}$ and the transverse fields $B_{i}$ are independent random variables with probability distributions $P_{I}(J)$ and $R_{I}(B)$, respectively. Both the $J_{i}$ and the $B_{i}$ are restricted to positive values. ${ }^{21}$

The qualitative features of the zero-temperature (ground state) phase diagram of the transverse-field Ising chain are easily discussed. If the typical interactions are much larger than the typical transverse fields, $J \gg B$, the ground state displays ferromagnetic long-range order in the $z$ direction. In the opposite limit, $J \ll B$, the ground state is a (field-polarized) quantum paramagnet. These two phases are separated by a quantum

\footnotetext{
21 We denote the transverse field by $B$ to distinguish it from a possible longitudinal (conjugate to the order parameter) field $h$. However, in the literature on the random transverse-field Ising model, $h$ is often used for the transverse field.
} 
phase transition at $J \sim B$. In fact, it has been shown rigorously [40] that the transition occurs when $\prod_{i} J_{i}=\prod_{i} B_{i}{ }^{22}$

Traditional approaches to investigating the quantum phase transition in the random transverse-field Ising chain would start by solving the clean problem, $J_{i} \equiv J, B_{i} \equiv B$. The randomness would then be treated in a perturbative fashion, maybe using the replica trick to carry out the disorder average. Such approaches have been successful for classical (thermal) phase transitions in disordered systems. However, they work very poorly for the quantum phase transition in the random-transverse field Ising model and many other quantum phase transitions. ${ }^{23}$ Later, we will understand the deeper reasons for this; basically, disorder effects turn out to be much stronger at quantum phase transitions than at classical ones.

In the next subsection we therefore introduce an alternative method that makes use of the disorder from the outset rather than treating it as a small perturbation of a clean system.

\subsection{Renormalizing the random transverse-field Ising chain}

\section{Basic idea}

The strong-disorder renormalization group was proposed in 1979 by Ma, Dasgupta, and $\mathrm{Hu}$ [43] to study random antiferromagnetic spin chains. It was greatly developed by D.S. Fisher in the mid 1990's [44, 45, 46] who pioneered its application to quantum phase transitions. Since then, the method has been employed in a broad variety of problems ranging from disordered quantum systems to classical nonequilibrium phase transitions (for a review, see, e.g., Ref. [3]).

The basic idea of the strong-disorder renormalization group consists in identifying the largest local energy scale in the entire system, i.e., the highest local excited state. As this excited state does not contribute to the low-energy physics important for the phase transition, it is integrated out, treating the neighboring energies (couplings) as perturbations. This works well if the disorder is strong [the distributions $P_{I}(J)$ and $R_{I}(B)$ are broad] because in this case, the largest local energy will be much larger than the neighboring ones. The method thus becomes controlled in the limit of strong disorder which gives the strong-disorder renormalization group its name.

The process of eliminating the largest local energy is now iterated; this gradually reduces the maximum energy in the system until the desired low-energy description of the phase transition physics is achieved.

\footnotetext{
22 The location of the quantum phase transition point in the random transverse-field Ising chain is fixed by the self-duality of the model: The form of the Hamiltonian is invariant under the duality transformation $\sigma_{i}^{x}=\mu_{i}^{z} \mu_{i+1}^{z} \sigma_{i}^{z}=\prod_{(j \leq i)} \mu_{j}^{x}$, (where $\mu^{x}$ and $\mu^{z}$ are the dual Pauli matrices). However, interactions and fields exchange their meaning, $J_{i} \rightleftarrows B_{i}$, under this transformation

23 Sometimes, perturbative methods show the disorder strength diverging and thus signal their own breakdown [41, 42].
} 

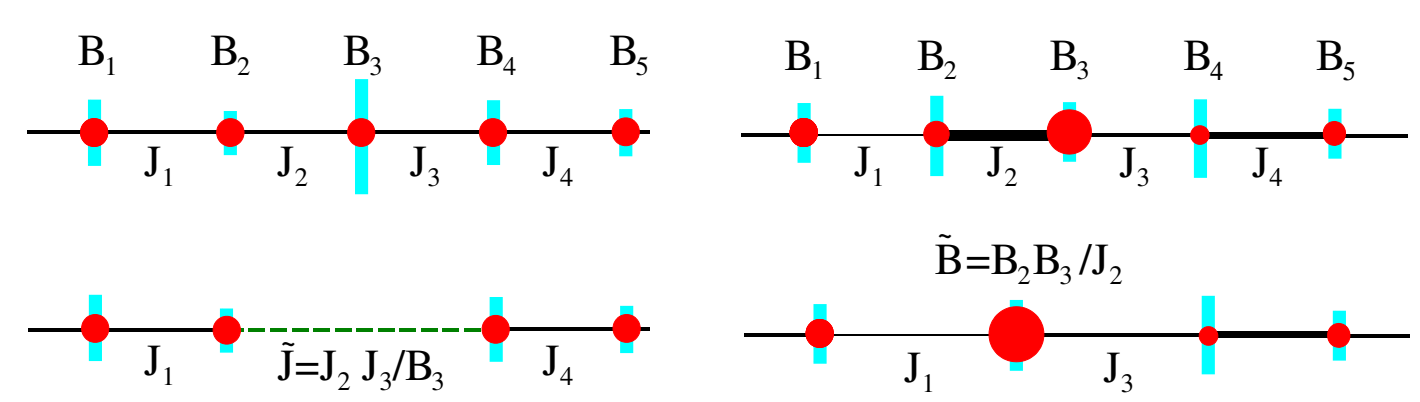

FIGURE 11. Strong disorder renormalization group steps. Left: strong transverse field (here $B_{3}$ ). The spin $\sigma_{3}$ is integrated out in second-order perturbation theory, generating an effective interaction $\tilde{J}$ between the neighboring spins $\sigma_{2}$ and $\sigma_{3}$. Right: strong interaction (here $J_{2}$ ). The spins $\sigma_{2}$ and $\sigma_{3}$ are parallel to each other. They can be represented by a single effective spin $\tilde{\sigma}$ in the transverse field $\tilde{B}$.

\section{Renormalization group recursions}

Let us implement the strong-disorder renormalization group for the random transverse-field Ising chain. The competing local energies in the Hamiltonian (27) are the interactions $J_{i}$ and the transverse fields $B_{i}$. We thus identify the largest local energy scale as $\Omega=\max \left(J_{i}, B_{i}\right)$. To integrate out the corresponding excited states, we need to distinguish $\Omega$ being an interaction and $\Omega$ being a field.

(i) If the largest local energy is a field, say $B_{3}$, the spin $\sigma_{3}$ is pinned in the positive $x$ direction (in the $\sigma^{x}$ eigenstate $|\rightarrow\rangle$ with eigenvalue +1 ). It does therefore not contribute to the $z$ magnetization and can be decimated (eliminated) from the system. However, virtual excitations of $\sigma_{3}$ from $|\rightarrow\rangle$ to $|\leftarrow\rangle$ generate an effective coupling $\tilde{J}$ between the neighboring spins $\sigma_{2}$ and $\sigma_{4} \cdot{ }^{24}$ To calculate $\tilde{J}$, we can consider the three-site system consisting of spins $\sigma_{2}, \sigma_{3}$, and $\sigma_{4}$ with Hamiltonian

$$
H=H_{0}+H_{1} \quad \text { with } \quad H_{0}=-B_{3} \sigma_{3}^{x}, \quad H_{1}=-J_{2} \sigma_{2}^{z} \sigma_{3}^{z}-J_{3} \sigma_{3}^{z} \sigma_{4}^{z} .
$$

Because $B_{3}>J_{2}, J_{3}$, we can treat $H_{1}$ in perturbation theory. In second order, we obtain

$$
\tilde{J}=J_{2} J_{3} / B_{3} .
$$

The new interaction $\tilde{J}$ is always smaller than either of the old ones. Thus, this renormalization step eliminates one degree of freedom (the spin $\sigma_{3}$ ) and reduces the maximum energy scale $\Omega$.

(ii) If the largest local energy is an interaction, say $J_{2}$, the spins $\sigma_{2}$ and $\sigma_{3}$ prefer to be parallel. The cluster consisting of $\sigma_{2}$ and $\sigma_{3}$ can thus be treated as a "superspin" $\tilde{\sigma}$ whose magnetic moment

$$
\tilde{\mu}=\mu_{2}+\mu_{3}
$$

is the sum of the moments associated with $\sigma_{2}$ and $\sigma_{3}$. This means that out of the four basis states of the cluster we keep the low-energy states $|\uparrow \uparrow\rangle$ and $|\downarrow \downarrow\rangle$ but not the high-

\footnotetext{
${ }^{24}$ If we simply eliminate $\sigma_{3}$ without taking the virtual excitations into account, the chain would be cut into two independent pieces because spins the $\sigma_{2}$ and $\sigma_{4}$ would not be coupled at all. Ferromagnetic long-range order would thus be impossible. This is clearly too rough an approximation.
} 
energy states $|\uparrow \downarrow\rangle$ and $|\downarrow \uparrow\rangle$. Virtual excitations to these high-energy states need to be taken into account to evaluate the influence of the transverse fields $B_{2}$ and $B_{3}$ on the effective spin $\tilde{\sigma}$. The effective transverse field can be calculated in perturbation theory for the two-site cluster consisting of $\sigma_{2}$ and $\sigma_{3}$ with

$$
H=H_{0}+H_{1} \quad \text { with } \quad H_{0}=-J_{2} \sigma_{2}^{z} \sigma_{3}^{z}, \quad H_{1}=-B_{2} \sigma_{2}^{x}-B_{3} \sigma_{3}^{x} .
$$

In second order in the transverse fields, we obtain

$$
\tilde{B}=B_{2} B_{3} / J_{2}
$$

The new transverse field $\tilde{B}$ is always smaller than either of the old ones.

The entire renormalization group step is summarized in Fig. 11. In both possible cases, decimation of a site or decimation of an interaction, one degree of freedom is eliminated and the maximum energy scale $\Omega$ is reduced. Otherwise, the structure of the Hamiltonian (27) is exactly preserved. Notice the symmetry between the recursion (29) for the interactions and the recursion (32) for the transverse fields. It reflects the self-duality of the Hamiltonian. We also note that the recursion (30) for the moments is additive while the recursion (32) for the fields is multiplicative, suggesting an exponential relation, $B \sim \exp (-c \mu)$ between the energy and the size of a cluster. This is an important observation that we will return to repeatedly in the coming sections.

\section{Flow equations}

The strong-disorder renormalization group method proceeds by iterating the above renormalization group steps, thus gradually decreasing the maximum energy $\Omega$ to zero. How do the interactions $J_{i}$ and transverse fields $B_{i}$ behave under this procedure? As the $J_{i}$ and $B_{i}$ are random quantities, we need to analyze the evolution of their probability distributions $P(J ; \Omega)$ and $R(B ; \Omega)$ with $\Omega$, starting from the initial (bare) distributions $P_{I}(J)$ and $R_{I}(B)$.

To derive the renormalization group flow equations for $P(J ; \Omega)$ and $R(B ; \Omega)$, assume that we reduce the maximum energy from $\Omega$ to $\Omega-d \Omega$ by decimating all interactions and fields in the energy interval $[\Omega-d \Omega, \Omega]$. As a result, the distribution $P(J ; \Omega)$ changes as

$$
\begin{aligned}
-d P(J ; \Omega)= & d \Omega R(\Omega ; \Omega)\left[-2 P(J ; \Omega)+\int d J_{1} d J_{2} P\left(J_{1} ; \Omega\right) P\left(J_{2} ; \Omega\right) \delta\left(J-J_{1} J_{2} / \Omega\right)\right] \\
& +d \Omega[R(\Omega ; \Omega)+P(\Omega ; \Omega)] P(J ; \Omega) .
\end{aligned}
$$

The terms in the first line are due to the decimation of strong transverse fields. The probability for such a decimation is given by the probability for finding a field within $d \Omega$ of the upper cutoff $\Omega$; it is identical to $d \Omega R(\Omega ; \Omega)$. Each such decimation removes two interactions from the system and introduces one new renormalized interaction given by the recursion (29). This is encoded in the terms inside the large bracket. As every decimation (of an interaction or field) reduces the number of remaining interactions by 
one, the normalization of the distribution $P(J ; \Omega)$ would change. The terms in the second line of (33) compensate for that and keep $P(J ; \Omega)$ normalized. ${ }^{25}$

The change of the field distribution $R(B ; \Omega)$ can be found along the same lines. We thus arrive at the renormalization group flow equations

$$
\begin{aligned}
-\frac{\partial P}{\partial \Omega} & =\left[P_{\Omega}-R_{\Omega}\right] P+R_{\Omega} \int d J_{1} d J_{2} P\left(J_{1} ; \Omega\right) P\left(J_{2} ; \Omega\right) \delta\left(J-J_{1} J_{2} / \Omega\right) \\
-\frac{\partial R}{\partial \Omega} & =\left[R_{\Omega}-P_{\Omega}\right] R+P_{\Omega} \int d B_{1} B h_{2} R\left(B_{1} ; \Omega\right) R\left(B_{2} ; \Omega\right) \delta\left(B-B_{1} B_{2} / \Omega\right)
\end{aligned}
$$

where $P$ and $R$ stand for $P(J ; \Omega)$ and $R(B ; \Omega)$ while $P_{\Omega}$ and $R_{\Omega}$ stand for $P(\Omega ; \Omega)$ and $R(\Omega ; \Omega)$, respectively. The solutions of these integro-differential equations in the limit $\Omega \rightarrow 0$ govern the low-energy physics of our system.

Before we start looking for solutions of the flow equations (34) and (35), let us change to more suitable variables. The multiplicative structure of the recursions for $J$ and $B$ suggests using logarithmic variables. We therefore introduce a logarithmic measure of the cutoff energy scale,

$$
\Gamma=\ln \left(\Omega_{I} / \Omega\right)
$$

where $\Omega_{I}$ is the initial (bare) value of the cutoff. We also define logarithmic variables for the interactions and fields by means of

$$
\zeta=\ln (\Omega / J), \quad \beta=\ln (\Omega / B) .
$$

The probability distributions $\bar{P}(\zeta ; \Gamma)$ and $\bar{R}(\beta ; \Gamma)$ of these logarithmic variables are related to the distributions of $J$ and $h$ via the transformations $P(J ; \Omega)=\bar{P}(\zeta ; \Gamma) / J$ and $R(B ; \Omega)=\bar{R}(\beta ; \Gamma) / B$. In the following, we are going to drop the bar over the new distributions as long as it is clear which distribution is meant.

By inserting the definitions (36) and (37) into (34) and (35), we can rewrite the flow equations in terms of the logarithmic variables. This gives

$$
\begin{aligned}
& \frac{\partial P}{\partial \Gamma}=\frac{\partial P}{\partial \zeta}+\left[P_{0}-R_{0}\right] P+R_{0} \int_{0}^{\zeta} d \zeta_{1} P\left(\zeta_{1} ; \Gamma\right) P\left(\zeta-\zeta_{1} ; \Gamma\right) \\
& \frac{\partial R}{\partial \Gamma}=\frac{\partial R}{\partial \beta}+\left[R_{0}-P_{0}\right] R+P_{0} \int_{0}^{\beta} d \beta_{1} R\left(\beta_{1} ; \Gamma\right) R\left(\beta-\beta_{1} ; \Gamma\right) .
\end{aligned}
$$

Here, $P$ and $R$ stand for $P(\zeta ; \Gamma)$ and $R(\beta ; \Gamma)$ while $P_{0}$ and $R_{0}$ denote $P(0 ; \Gamma)$ and $R(0 ; \Gamma)$.

How can one solve these flow equations? D. S. Fisher provided an essentially complete analysis in two long papers [45, 46]. It requires significant mathematical effort and is beyond the scope of these lectures. Instead, we are going to use an ansatz for the distributions $P$ and $R$ that will lead us to the correct fixed point solutions. ${ }^{26}$ Specifically, let

\footnotetext{
25 The overall minus sign in front of $d P(J ; \Omega)$ stems from the fact that $d \Omega$ changes the cutoff $\Omega$ downwards.

26 This approach does not guarantee, of course, that there are no other solutions that are important for the physics of our problem. To address this question one needs to consult the complete solution.
} 
us assume that both distributions are simple exponentials,

$$
P(\zeta ; \Gamma)=P_{0}(\Gamma) \exp \left[-P_{0}(\Gamma) \zeta\right], \quad R(\beta ; \Gamma)=R_{0}(\Gamma) \exp \left[-R_{0}(\Gamma) \beta\right]
$$

with cutoff-dependent inverse widths $P_{0}$ and $R_{0}$. Inserting this ansatz into (38) and (39), we find that the distributions indeed fulfill the flow equations provided that the coefficients $P_{0}$ and $R_{0}$ are solutions of the coupled differential equations

$$
\begin{aligned}
& \frac{d P_{0}}{d \Gamma}=-R_{0} P_{0}, \\
& \frac{d R_{0}}{d \Gamma}=-R_{0} P_{0} .
\end{aligned}
$$

We thus have turned the flow equations for the probability distributions $P(\zeta ; \Gamma)$ and $R(\beta ; \Gamma)$ into flow equations for the coefficients $P_{0}$ and $R_{0}$ which can be solved much more easily.

In the following subsection, we will look for solutions of these flow equations and analyze their behavior in the low-energy limit $\Gamma \rightarrow \infty$.

\subsection{Infinite-randomness scenario}

\section{Critical point}

According to Pfeuty's exact result [40], the random transverse-field Ising model is critical when $\prod_{i} J_{i}=\prod_{i} B_{i}$. At the critical point, the coefficients $P_{0}(\Gamma)$ and $R_{0}(\Gamma)$ must therefore be identical. This also follows from the self-duality of the Hamiltonian discussed in Sec. 3.1. The two flow equations (41) and (42) now coincide and read

$$
\frac{d P_{0}}{d \Gamma}=-P_{0}^{2}
$$

This differential equation can be easily integrated, giving $P_{0}=1 /\left(\Gamma-\Gamma_{0}\right)$. The integration constant $\Gamma_{0}$ can be dropped as it just amounts to a redefinition of the reference energy scale $\Omega_{I}$. We thus arrive at the "fixed point" solution ${ }^{27}$

$$
P(\zeta ; \Gamma)=\frac{1}{\Gamma} e^{-\zeta / \Gamma}, \quad R(\beta ; \Gamma)=\frac{1}{\Gamma} e^{-\beta / \Gamma}
$$

In the low-energy limit, $\Gamma \rightarrow \infty$, these distributions become arbitrarily broad implying that the randomness in the system becomes arbitrarily strong. For this reason, the resulting critical point is called an infinite-randomness critical point. In terms of the

\footnotetext{
27 This solution is a fixed point in the sense that its functional form does not change with $\Gamma \rightarrow \infty$. If we rescale $\zeta$ and $\beta$ by $\Gamma$, the resulting distributions become true stationary points of the renormalization group flow.
} 
original variables $J$ and $B$, the distributions are highly singular,

$$
P(J ; \Omega)=\frac{1}{\Gamma J}\left(\frac{J}{\Omega}\right)^{1 / \Gamma}, \quad R(B ; \Omega)=\frac{1}{\Gamma B}\left(\frac{B}{\Omega}\right)^{1 / \Gamma} .
$$

Let us discuss some of the key properties of the critical fixed point solution (44) in more detail. We start by analyzing the number $n_{\Omega}$ of sites (clusters) that survive in the renormalized random transverse-field Ising chain at some energy scale $\Omega$. Every renormalization group step reduces the number of clusters by one, either because a cluster is decimated (for a large transverse field) or because two clusters are combined (for a large interaction). The number of clusters thus changes by $d n_{\Omega}=-n_{\Omega}\left(P_{\Omega}+\right.$ $\left.R_{\Omega}\right) d \Omega$ as the cutoff is reduced from $\Omega$ to $\Omega-d \Omega$. Transforming to logarithmic variables leads to

$$
\frac{d n_{\Gamma}}{d \Gamma}=-\left(P_{0}+R_{0}\right) n_{\Gamma}=-\frac{2}{\Gamma} n_{\Gamma}
$$

Integrating this differential equation shows that the number of clusters decreases as

$$
n_{\Gamma} \sim \Gamma^{-2} \quad \text { or } \quad n_{\Omega} \sim\left[\ln \left(\Omega_{I} / \Omega\right)\right]^{-2} .
$$

The typical distance $\ell(\Omega)$ between the surviving clusters is inversely proportional to their number, yielding

$$
\ln \left(\Omega_{I} / \Omega\right) \sim[\ell(\Omega)]^{\psi} \quad \text { with } \quad \psi=1 / 2 .
$$

The self-duality of the critical solution implies that $\ell(\Omega)$ gives not just the typical distance between clusters but also the typical length of a cluster.

We note that the dependence of the energy scale on the length scale established by (48) is exponential and so is the dependence of the time scale on the length scale. This differs from the power-law dependence (2) found at conventional (clean) critical points. An exponential relation between lengths and times is called activated or tunneling dynamical scaling (as opposed to the usual power-law dynamical scaling) and $\psi$ is called the tunneling exponent. It also means that the dynamical exponent $z$ is formally infinite.

In addition to the number of surviving clusters, one can also calculate their magnetic moment $\mu_{\Omega}$ (i.e., the number of original sites represented by an effective spin). The calculation is rather lengthy [46]; we therefore simply quote the result

$$
\mu_{\Omega} \sim\left[\ln \left(\Omega_{I} / \Omega\right)\right]^{\phi}
$$

where the exponent $\phi=(\sqrt{5}+1) / 2$ is given by the golden mean. ${ }^{28}$ These are highly unusual properties that are reflected in unusual behaviors of observables at the infiniterandomness critical point.

\footnotetext{
28 The fact that the exponent $\phi$ which governs the magnetic moment is smaller than the exponent 2 which governs the length of a cluster suggests that the surviving clusters have a fractal structure with lots of holes.
} 
The fact the probability distributions of the fields and interactions become infinitely broad as the critical fixed point is approached implies that the method becomes asymptotically exact because the perturbative treatment of the recursion steps becomes better and better. This also means that the above critical exponent values are exact which is remarkable because exact exponent values are known for very few phase transitions only. Moreover, the infinite-randomness character of the critical point explains why perturbative methods fail to capture the physics of this problem. Even if the bare disorder strength is a small, the disorder becomes arbitrarily strong on large length scales.

\section{Off-critical solutions}

Right at the critical point, the two decimation processes (eliminating clusters with strong transverse fields and building larger clusters by decimating a strong interaction) balance each other out such that $P_{0}=R_{0}$ in the low-energy limit. In contrast, off criticality, one of the processes wins.

On the paramagnetic side of the transition defined by $\prod_{i} B_{i}>\prod_{i} J_{i}$ or, equivalently, $[\ln B]_{d i s}>[\ln J]_{d i s}$, the building of clusters essentially stops at some value of the cutoff $\Omega$ because most $B$ are larger than most $J$. From then on, only transverse fields are decimated, producing smaller and smaller interactions. Thus, we expect the field distribution $R(\beta)$ to become stationary with $\Omega \rightarrow 0$ while $P(\zeta)$ should rapidly shift to small interactions (large $\zeta$ ). On the ferromagnetic side, we expect the opposite behavior, i.e., the elimination of clusters stops below some value of the cutoff $\Omega$. Here, $P(\zeta)$ should become stationary and $R(\beta)$ should rapidly move to large $\beta$.

In the following, we focus on the paramagnetic side. Off criticality, $P_{0} \neq R_{0}$, thus the two flow equations (41) and (42) need to be solved together. By subtracting them from each other, we obtain $(d / d \Gamma)\left[R_{0}-P_{0}\right]=0$ which yields

$$
R_{0}(\Gamma)=P_{0}(\Gamma)+2 \delta
$$

where $\delta$ is an energy-independent constant. What is the physical meaning of $\delta$ ? Note that $[\ln (h / \Omega)]_{\text {dis }}=[-\beta]_{\text {dis }}=-1 / R_{0}$ and $[\ln (J / \Omega)]_{\text {dis }}=[-\zeta]_{\text {dis }}=-1 / P_{0}$. Thus $[\ln h]_{\text {dis }}-[\ln J]_{\text {dis }}=1 / P_{0}-1 / R_{0} \sim R_{0}-P_{0}=2 \delta$ for small $\delta$. We conclude that $\delta$ is a measure of the distance from criticality.

After inserting (50), the flow equations (41) and (42) can be easily solved. We find

$$
P_{0}=2 \delta /[\exp (2 \delta \Gamma)-1] \text {. }
$$

In the low-energy limit, $\Gamma \rightarrow \infty$, this results in the fixed-point distributions

$$
\begin{aligned}
& R(\beta)=2 \delta \exp [-2 \delta \beta] \\
& P(\zeta)=2 \delta e^{-2 \delta \Gamma} \exp \left[-2 \delta e^{-2 \delta \Gamma} \beta\right] .
\end{aligned}
$$

As expected from our qualitative discussion above, the field-distribution $R(\beta)$ is indeed independent of $\Gamma$ while the interaction distribution $P(\zeta)$ rapidly becomes extremely broad. This means, almost all interactions $J$ are extremely small, and the surviving 
clusters decouple. Transforming (52) to the original transverse fields $B$ yields a powerlaw distribution

$$
R(B ; \Omega)=\frac{2 \delta}{B}\left(\frac{B}{\Omega}\right)^{2 \delta}
$$

characterized by a nonuniversal exponent that changes with distance $\delta$ from the critical point.

The number of clusters surviving at energy scale $\Gamma$ can be determined from the differential equation $\left(d n_{\Gamma}\right) /(d \Gamma)=-\left(R_{0}+P_{0}\right) n_{\Gamma}$, as at the critical point. This gives $n_{\Gamma}=n_{0} \exp (-2 \delta \Gamma)$ or, equivalently, $n_{\Omega} \sim\left(\Omega / \Omega_{I}\right)^{2 \delta}$. The typical distance $\ell(\Omega)$ between clusters surviving at energy scale $\Omega$ therefore behaves as

$$
\ell(\Omega) \sim 1 / n_{\Omega} \sim\left(\Omega / \Omega_{I}\right)^{-2 \delta} .
$$

In contrast to the activated dynamical scaling (48) at the critical point, this is a power-law relation characterized by a nonuniversal dynamical exponent $z^{\prime}=1 /(2 \delta)$.

To find the correlation length and time at a given distance $\delta$ from criticality we can calculate the crossover energy scale $\Gamma_{x}$, beyond which the off-critical flow (51) appreciably deviates from the critical flow $P_{0}=1 / \Gamma$. This gives $\Gamma_{x} \sim 1 /(2 \delta)$. According to (47), the characteristic length is $\ell\left(\Gamma_{x}\right) \sim \Gamma_{x}^{2} \sim 1 /(2 \delta)^{2}$ at this energy scale. We conclude that the correlation length exponent takes the value $v=2$. Interestingly, this means that it exactly saturates the inequality $d v \geq 2$ derived by Chayes et al. [33].

\section{Thermodynamic observables}

The general strategy for finding the temperature dependencies of observables within the strong-disorder renormalization group approach consists in running the renormalization group until the cutoff reaches the value $\Omega=k_{B} T$. Close to criticality and at low energies $\Omega$, the probability distributions of $J$ and $B$ become very broad. This means, all degrees of freedom eliminated during this process have energies much larger than $k_{B} T$ and do not contribute to the thermodynamics. In contrast, all the remaining interactions and transverse fields are much smaller than $k_{B} T$. The remaining degrees of freedom can thus be considered as free. Consequently, observables are simply the sums over independent contributions from the surviving clusters.

Let us apply this strategy to the thermodynamics right at criticality. According to (47) and (49), the number of surviving clusters at energy scale $\Omega$ behaves as $n_{\Omega} \sim$ $\left[\ln \left(\Omega_{I} / \Omega\right]^{-1 / \psi}\right.$ with $\psi=1 / 2$ while the typical moment of such a cluster scales as $\mu_{\Omega} \sim\left[\ln \left(\Omega_{I} / \Omega\right]^{\phi}\right.$ with $\phi=(\sqrt{5}-1) / 2$. Each cluster (being effectively free) contributes a Curie term $\mu^{2} / T$ to the magnetic susceptibility. The total susceptibility thus reads

$$
\chi(T)=\frac{1}{T} n_{T} \mu_{T}^{2} \sim \frac{1}{T}\left[\ln \left(\Omega_{I} / k_{B} T\right)\right]^{2 \phi-1 / \psi} .
$$

To estimate the entropy, we note that each surviving cluster is equivalent to a two-level system and contributes $k_{B} \ln (2)$ to the entropy. The total entropy is thus given by

$$
S(T)=k_{B} \ln (2) n_{T} \sim k_{B}\left[\ln \left(\Omega_{I} / k_{B} T\right)\right]^{-1 / \psi} .
$$


The specific heat can be calculated by taking the appropriate temperature derivative of the entropy. This yields

$$
C_{V}(T)=T(\partial S / \partial T)=(\partial S / \partial \ln (T)) \sim k_{B}\left[\ln \left(\Omega_{I} / k_{B} T\right)\right]^{-1 / \psi-1} .
$$

All these quantities display unusual logarithmic temperature dependencies which stem from the activated dynamical scaling (48).

The same strategy for determining observables can also be applied off criticality. According to (55), the number of surviving clusters on the paramagnetic side of the transition scales as $n_{\Omega} \sim \Omega^{2 \delta}=\Omega^{1 / z^{\prime}}$ with the energy cutoff $\Omega$. This results in a total entropy of

$$
S(T)=k_{B} \ln (2) n_{T} \sim T^{1 / z^{\prime}}
$$

and a specific heat of

$$
C_{V}(T)=T(\partial S / \partial T) \sim T^{1 / z^{\prime}}
$$

The magnetic susceptibility can be found along the same lines, yielding

$$
\chi(T)=\frac{1}{T} n_{T} \mu_{T}^{2} \sim T^{1 / z^{\prime}-1} .
$$

Note that the magnetic moment of a surviving cluster on the paramagnetic side of the transition increases only very slowly (logarithmically) with decreasing energy cutoff $\Omega$ because almost all decimations are eliminations of sites [46]. Therefore, $\mu_{T}$ does not contribute to the leading temperature dependence of the susceptibility.

Equations (59) to (61) show that the thermodynamic behavior of the random transverse-field Ising chain is highly singular not just right at the critical point but also in its vicinity. These off-critical singularities are examples of the celebrated Griffiths singularities that we will study from a more general perspective in Sec. 4.

\section{GRIFFITHS SINGULARITIES AND THE GRIFFITHS PHASE}

When a clean system undergoes a phase transition, thermodynamic quantities are singular right at the transition point. Away from the transition point, they are generally nonsingular. However, at the end of the last section, we have seen that the random transversefield Ising model features singular thermodynamics not just at the critical point but also in its vicinity. It turns out that such off-critical singularities generically occur at phase transitions in disordered systems. They are called the Griffiths singularities after R. B. Griffiths who first proved their existence in 1969 [47].

In this section, we will develop a general qualitative understanding of the physics leading to the Griffiths singularities; and we will discuss their consequences for classical and quantum phase transitions.

\subsection{Rare regions}

Let us start from a clean classical ferromagnet given by an Ising model on a cubic lattice, $H=-J \sum_{\langle i j\rangle} S_{i} S_{j}$. It undergoes a continuous phase transition from a paramagnet 


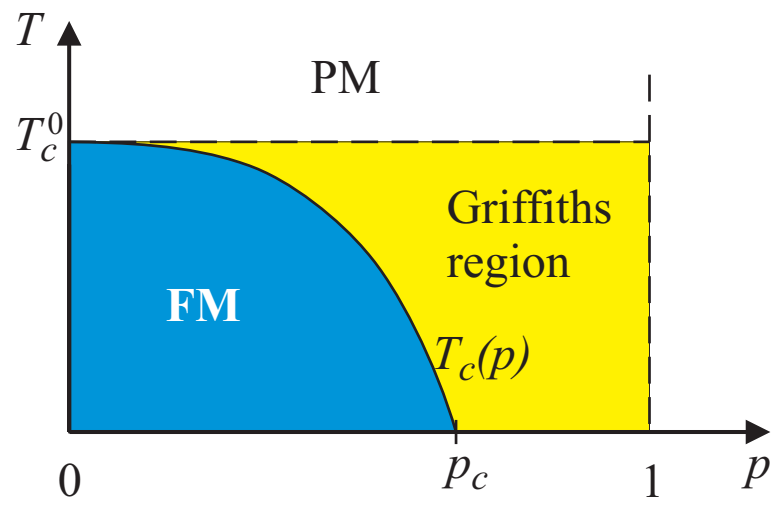

FIGURE 12. Schematic temperature-dilution phase diagram of the site-diluted Ising model (62). With increasing dilution, the critical temperature $T_{c}(p)$ is suppressed, it vanishes at the percolation threshold $p_{c}$. The Griffiths region (or Griffiths phase) comprises the part of the paramagnetic phase below $T_{c}^{0}$ where locally ordered "clean" clusters can exist.

to a ferromagnet at the clean critical temperature $T_{c}^{0}$. We now introduce quenched disorder by randomly removing spins from the lattice. The Hamiltonian of the resulting site-diluted Ising model reads

$$
H=-J \sum_{\langle i j\rangle} \varepsilon_{i} \varepsilon_{j} S_{i} S_{j}
$$

where the quenched random variables $\varepsilon_{i}$ can take the values 0 (representing a vacancy) or 1 (representing a spin) with probabilities $p$ and $1-p$, respectively.

As the dilution reduces the overall tendency towards ferromagnetism, the critical temperature $T_{c}(p)$ of the diluted system will decrease with increasing $p$ (see Fig. 12). It vanishes at the percolation threshold $p_{c}$ of the lattice because ferromagnetic long-range order is impossible if the lattice consists of disconnected finite-size clusters only.

Due to statistical fluctuations in the vacancy distribution, large vacancy-free spatial regions can exist even for high vacancy concentrations (with a very small but nonzero probability). As such rare regions are finite-size pieces of the clean system, their spins align parallel to each other below the clean critical temperature $T_{c}^{0}$. Because they are of finite size, these regions cannot undergo a true phase transition by themselves, but for temperatures between $T_{c}^{0}$ and the actual transition temperature $T_{c}(p)$, they act as large superspins.

The parameter region where such locally ordered rare regions exist, but long-range ordered has not yet developed is called the Griffiths phase. (More appropriately, it should be called the Griffiths region as it is part of the paramagnetic phase.) In our example the Griffiths region comprises the area between $T_{c}^{0}$ and $T_{c}(p)$ in the temperature-dilution plane.

So far, we have focused on the paramagnetic side of the transition. How can we generalize the idea of a Griffiths region or Griffiths phase to the ferromagnetic side? The simplest idea seems to be to consider rare "holes" in the magnetic order, i.e., rare vacancy-rich regions in a magnetically ordered bulk. However, in contrast to the locally ordered rare regions considered above whose magnetization can fluctuate between up 

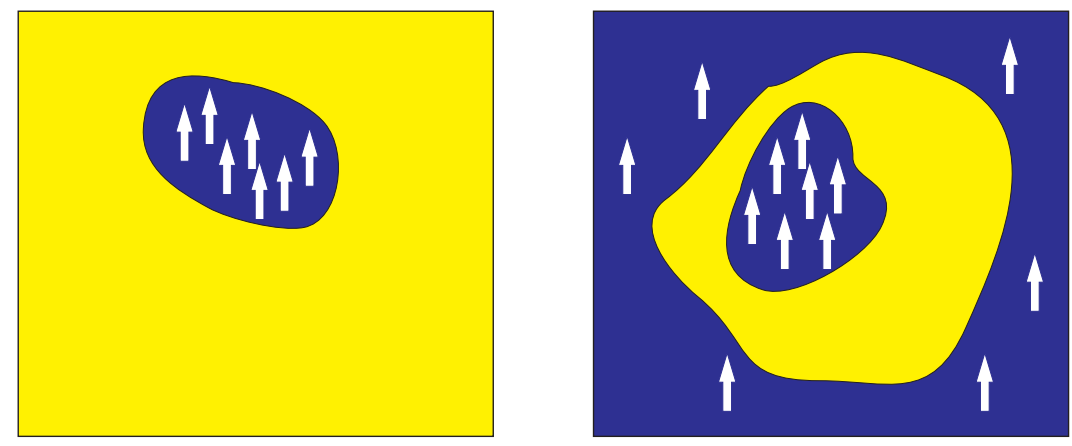

FIGURE 13. Left: The paramagnetic Griffiths phase is due to rare locally ferromagnetic regions embedded in the paramagnetic bulk. Right: The ferromagnetic Griffiths phase is caused by locally ferromagnetic regions located inside paramagnetic "holes" in the bulk ferromagnet.

and down, holes do not have an associated low-energy degree of freedom. Instead of simple "holes" one should thus consider locally ordered island inside holes. Such islands can fluctuate between up and down because they are only very weakly coupled to the bulk ferromagnet outside the hole (see Fig. 13). This conceptual difference will be responsible for slight differences in the resulting Griffiths singularities on the two sides of the transition. In our example of a site-diluted Ising model, the ferromagnetic Griffiths phase comprises all of the ferromagnetic phase for $p>0$.

Note that the precise location and extension of the Griffiths phases depend on the details of the system at hand. For example, we could study an Ising model with bond (interaction) randomness instead of the site-diluted Ising model (62). It is given by the Hamiltonian

$$
H=-\sum_{\langle i j\rangle} J_{i j} S_{i} S_{j}
$$

in which the nearest neighbor interactions $J_{i j}$ can take the values $J_{A}$ and $J_{B}>J_{A}$ with probability $p$ and $1-p$, respectively. In this model, the paramagnetic and ferromagnetic Griffiths phases are both located between the transition temperature $T_{c}^{B}$ of a system in which all bonds have value $J_{B}$ and the transition temperature $T_{c}^{A}$ of a system with all bonds having value $J_{A}$ (see Fig. 14).

Why are these rare large locally ordered regions interesting? In the next subsections, we will see that, despite being rare, they are responsible for the peculiar Griffiths singularities in the vicinity of a phase transition in a disordered system.

\subsection{Classical Griffiths singularities}

\section{Thermodynamics of the Griffiths phase}

In 1969, Griffiths [47] showed that the free energy is a singular function of the external control parameters everywhere in the Griffiths phase. The physical origin of the singular behavior was later identified as being the contribution of the rare regions to the 


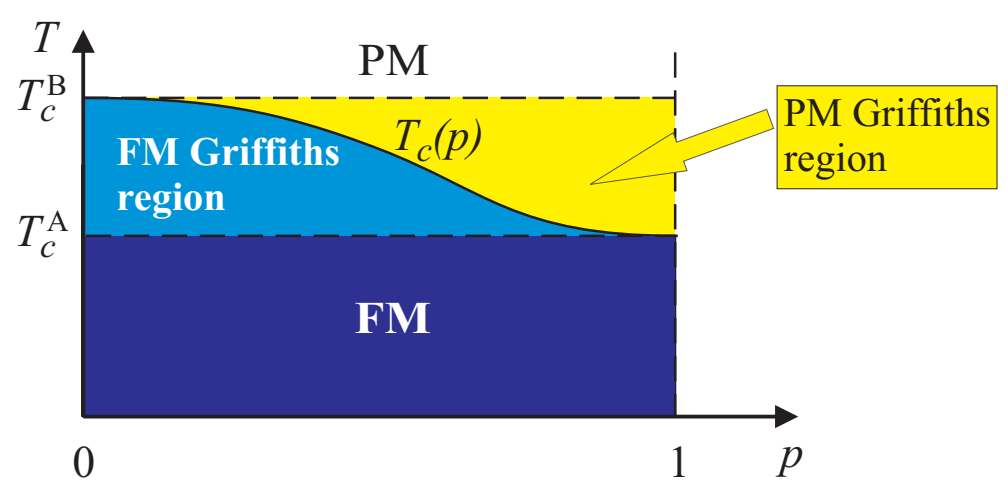

FIGURE 14. Phase diagram of the random-bond Ising model (63). The Griffiths phases are located between the transition temperatures $T_{c}^{A}$ and $T_{c}^{B}$ of systems with uniform interactions $J_{A}$ or $J_{B}$, respectively. The conventional paramagnetic phase is located above $T_{c}^{B}$, and the conventional ferromagnet is below $T_{c}^{A}$

thermodynamic behavior. To understand this mechanism, let us estimate the rare region contribution to the magnetization-field curve $m(h)$ and to the magnetic susceptibility $\chi$.

If a weak magnetic field $h$ is applied to a disordered magnet in the (paramagnetic) Griffiths phase, the locally ordered rare regions act as large superspins whose magnetic moment is proportional to their volume, $\mu\left(L_{R R}\right) \sim \mu_{B} V \sim L_{R R}^{d}$, where $L_{R R}$ is the linear size of the rare region. The energy gain due to aligning the moment with the external field can be estimated as $\Delta E=-h \mu \sim h \mu_{B} V$. If $|\Delta E|>k_{B} T$, the superspin is essentially fully polarized in field direction. For $|\Delta E|<k_{B} T$, its magnetization is small and governed by linear response.

The singular contribution of the large rare regions to the magnetization-field curve can therefore be estimated by summing over all rare regions with $|\Delta E|>k_{B} T$. This gives

$$
m_{R R}(h) \approx \sum_{|\Delta E|>k_{B} T} w\left(L_{R R}\right) \mu\left(L_{R R}\right)
$$

where $w\left(L_{R R}\right)$ is the probability for finding a locally ordered rare region of linear size $L_{R R}$. Basic combinatorics yields that the probability for finding a large spatial region devoid of impurities decays exponentially with its volume,

$$
w\left(L_{R R}\right) \sim \exp \left(-\tilde{p} L_{R R}^{d}\right) .
$$

For the diluted ferromagnet (62), the constant $\tilde{p} \sim-\ln (1-p)$. To exponential accuracy, the rare region magnetization can now be estimated as

$$
m_{R R}(h) \sim \int_{L_{x}}^{\infty} d L_{R R} \exp \left(-\tilde{p} L_{R R}^{d}\right) \mu_{B} L_{R R}^{d}
$$

where the integral is over all rare regions larger than a critical size $L_{x}$ defined by $L_{x}^{d} \sim k_{B} T /\left(\mu_{B} h\right)$. This results in

$$
m_{R R}(h) \sim \exp \left[-\tilde{p} k_{B} T /\left(\mu_{B} h\right)\right]
$$


The rare region magnetization is thus indeed a singular function of the applied field $h$, as required by Griffiths' proof. However, the singularity is an essential singularity leading to an extremely small contribution.

The magnetic susceptibility $\chi$ can be analyzed similarly. Each locally ordered rare region makes a Curie contribution $\mu^{2}\left(L_{R R}\right) / T$ to $\chi$. The total rare region susceptibility can therefore be estimated as

$$
\chi_{R R}(T) \sim \int d L_{R R} w\left(L_{R R}\right) \mu_{B}^{2} L_{R R}^{2 d} / T
$$

This equation shows that the susceptibility of an individual rare regions does not increase fast enough to overcome the exponential decay of the rare region probability $w$ with increasing size $L_{R R}$. Consequently, large rare regions only make an exponentially small contribution to the susceptibility.

Analogous estimates can also be performed in the ferromagnetic Griffiths phase on the ordered side of the phase transition. The main difference between the paramagnetic and ferromagnetic Griffiths phases is in the probability for finding a rare region. On the paramagnetic side, the rare event is finding a vacancy-free region of linear size $L_{R R}$ which leads to (65). On the ferromagnetic side, the rare event is finding a large enough vacancy-rich region around a locally ordered island (see Fig. 13). The required volume of this "shell" scales as $L_{R R}^{d-1} \xi_{B}$ where $\xi_{B}$ is the bulk correlation length in this vacancyrich region. Thus the probability of finding such a "island in a hole" behaves as

$$
w_{F M}\left(L_{R R}\right) \sim \exp \left(-c L_{R R}^{d-1}\right)
$$

The overall conclusion of these considerations is that classical rare region phenomena are very weak because the singularity in the free energy is only an essential one [48, 49, $50,51]$. To the best of our knowledge, classical Griffiths singularities in thermodynamic quantities have therefore not been verified in experiment. ${ }^{29}$

\section{Dynamical Griffiths singularities}

In contrast to the thermodynamics discussed above, the long-time dynamics in a classical Griffiths phase is dominated by the rare regions.

The classical Ising Hamiltonians (62) and (63) do not have any internal dynamics. However, we can add a heuristic dynamics to these models. The simplest case is a purely relaxational dynamics which corresponds to the so-called model A in the famous classification of Hohenberg and Halperin [52]. Microscopically, this type of dynamics is due to collisions with other degrees of freedom. In computer simulations, it can be realized via the Glauber [53] or Metropolis [54] algorithms.

29 This conclusion holds for uncorrelated or short-range correlated disorder only. We will see in Sec. 4.3 that long-range disorder correlations in space greatly enhance the rare region effects. 
One of the simplest quantities for studying the real-time dynamics is the spin autocorrelation function

$$
C(t)=\frac{1}{N} \sum_{i}\left\langle S_{i}(t) S_{i}(0)\right\rangle
$$

where $S_{i}(t)$ is the value of the spin at site $i$ and time $t$, the sum is over all $N$ sites of the lattice, and $\langle\ldots\rangle$ denotes the thermodynamic average. It is related to the Fourier transform of the local dynamic susceptibility.

The fluctuations associated with the rare regions are much slower than the bulk fluctuations in the paramagnetic Griffiths phase because they involve a coherent change of the magnetization in a large volume. As different rare regions are independent of each other, their contribution to the auto-correlation function can be estimated as

$$
C_{R R}(t) \sim \int d L_{R R} w\left(L_{R R}\right) L_{R R}^{d} \exp \left[-t / \xi_{t}\left(L_{R R}\right)\right]
$$

where $\xi_{t}\left(L_{R R}\right)$ is the correlation time (or life time) of a single rare region, i.e., the typical time it takes to reverse the rare region magnetization. How can we estimate this correlation time? The most effective way of reversing the rare region magnetization is to create a domain wall and then move it across the rare region. The energy cost of creating such a domain wall is given by $\sigma A_{D W}$ where $\sigma$ is a temperature-dependent constant and

$A_{D W}$ is the domain wall area. Thus, the domain wall energy scales as $\sigma L_{R R}^{d-1}$ with the rare region size. The creation of the domain wall is an activated process that happens at an exponentially small rate, implying

$$
\xi_{t}\left(L_{R R}\right) \sim \tau_{0} \exp \left[C \sigma L_{R R}^{d-1} /\left(k_{B} T\right)\right]
$$

where $C$ is some constant of order unity. After inserting the correlation time into the autocorrelation function (71), the integral can be estimated by means of the saddle point method. This yields

$$
C_{R R}(t) \sim \exp \left[-\tilde{C}(\ln t)^{d /(d-1)}\right]
$$

in the long-time limit ( $\tilde{C}$ is another constant). The long-time decay of the rare region autocorrelation function is much slower than the exponential decay of the bulk system off criticality. Thus, the long-time dynamics of the disordered Ising model is dominated by the rare regions $[55,56,57,58]$.

\subsection{Quantum Griffiths singularities}

\section{Rare region density of states}

In the last subsection we have analyzed the Griffiths singularities close to a classical phase transition and found them to be weak (at least in the case of thermodynamic quantities). Now, we are going to address the same question for a quantum phase transition. 


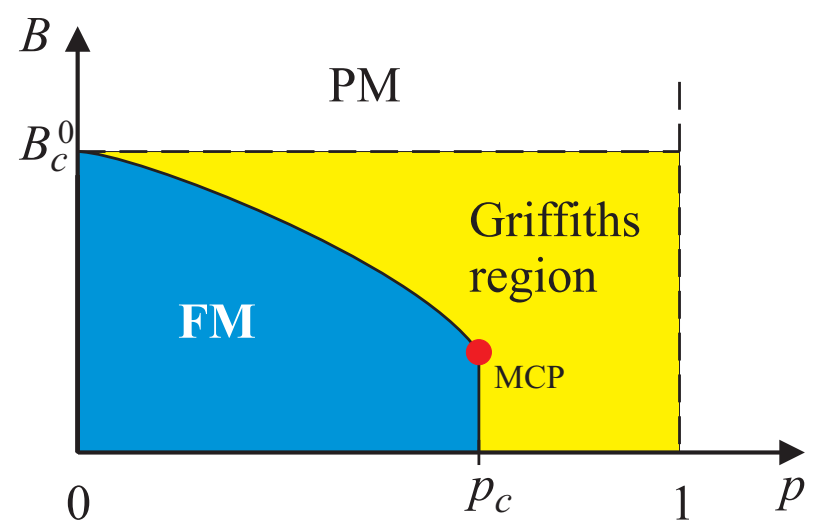

FIGURE 15. Schematic zero-temperature phase diagram of the site-diluted transverse-field Ising model (74). With increasing dilution, the critical field $B_{c}(p)$ is suppressed. A multicritical point (MCP) separates the generic quantum phase transition for $p<p_{c}$ from the percolation transition at $p_{c}$. Ferromagnetic longrange order is impossible for dilutions above $p_{c}$. The Griffiths region (or Griffiths phase) comprises the part of the paramagnetic phase below $B_{c}^{0}$ where locally ordered "clean" clusters can exist.

Our example is the three-dimensional transverse-field Ising model, and we introduce disorder via site-dilution. The Hamiltonian reads

$$
H=-J \sum_{\langle i j\rangle} \varepsilon_{i} \varepsilon_{j} \sigma_{i}^{z} \sigma_{j}^{z}-B \sum_{i} \varepsilon_{i} \sigma_{i}^{x}
$$

As in the last section, the $\varepsilon_{i}$ are quenched random variables that take values 0 or 1 with probabilities $p$ and $1-p$, respectively. Note that we again use $B$ for the transverse field to distinguish it from a longitudinal field $h$ in $z$-direction.

As was discussed in Sec. 1.2, the undiluted system undergoes a (zero-temperature) quantum phase transition from a ferromagnet to a paramagnet at a critical value $B_{c}^{0}$ of the transverse field. Site dilution reduces the tendency towards ferromagnetism and leads to a phase diagram similar to the classical case (with the transverse field playing the role of the temperature). It is shown in Fig. 15. The paramagnetic Griffiths region consists of the area between $B_{c}^{0}$ and the ferromagnetic phase boundary while the ferromagnetic Griffiths phase comprises the entire ordered phase.

In contrast to the classical case, the phase boundary $B_{c}(p)$ does not drop to zero field at the percolation threshold $p_{c}$ of the lattice. It rather ends at a multicritical point located at $p_{c}$ and some nonzero field $B_{\mathrm{MCP}}$. This implies that the site-diluted transverse field Ising model features two different quantum phase transitions, a generic transition at $p<p_{c}$, driven by quantum fluctuations and a quantum percolation transition at $p=p_{c}$ (across the vertical line in Fig. 15), driven by the lattice geometry. By universality, we expect the generic transition to show the same critical behavior as a three-dimensional version of the random transverse-field Ising model studied in Sec. 3. The percolation transition is in a different universality class which was investigated by Senthil and Sachdev [59].

Why are the classical and quantum phase diagrams (Figs. 12 and 15) different? An intuitive picture of the difference follows from the quantum-to-classical mapping. In the classical case, the critical temperature drops to zero at $p_{c}$ because the critical infinite percolation cluster contains so-called red sites. A red site is a site that joins two otherwise 


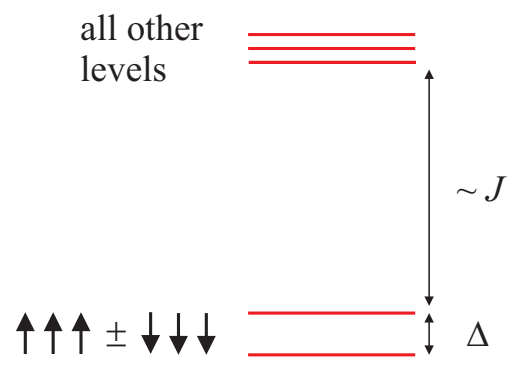

FIGURE 16. Schematic energy spectrum of a single rare region in the regime $B \ll B_{c}^{0}$. The two lowlying states are the symmetric and antisymmetric combinations of fully polarized "up" and "down" states. They are separated by a large gap of order $J$ from the rest of the spectrum.

disconnected subclusters. Forming a domain wall at such a red site costs a finite energy of $2 J$. The fluctuations of the relative magnetization orientations on the two subclusters destroy the long-range order. In the quantum case, the red sites turn into red lines that are infinitely long along the imaginary time direction. This suppresses the orientation fluctuations between the two subclusters. Long-range order at $p_{c}$ is therefore possible. A more detailed discussion is given, for example, in Ref. [60].

We now focus on the paramagnetic Griffiths phase and study the contributions of the rare regions to various observables. As in the classical case, we do this by combining the rare region probability $w\left(L_{R R}\right)$ with the properties of individual rare regions. The energy spectrum of a single locally ordered region in the regime $B \ll B_{c}^{0}$ is sketched in Fig. 16. For zero transverse field, $B=0$, the two fully polarized states $|\uparrow \uparrow \ldots \uparrow\rangle$ and $|\downarrow \downarrow \ldots \downarrow\rangle$ (all spins "up" or all "down") are the degenerate ground states of the rare region. They are separated from all other states by a large energy gap of order $J$. If a small field is switched on, the degeneracy is lifted by forming the symmetric and antisymmetric superpositions $|\uparrow \uparrow \ldots \uparrow\rangle \pm|\downarrow \downarrow \ldots \downarrow\rangle$ but the large gap to the other energy levels remains.

Since we are interested in the low-energy physics of the system, we can neglect all these high-energy states and treat the rare region as a two-level system. The value of the tunnel splitting $\Delta$ between the two low-lying states $|\uparrow \uparrow \ldots \uparrow\rangle \pm|\downarrow \downarrow \ldots \downarrow\rangle$ can be estimated using perturbation theory in $B$. In $n$-th order in $B$, this produces terms of the type $\left\langle\uparrow \uparrow \ldots \uparrow\left|\left(B \sum_{i} \sigma_{i}^{x}\right)^{n}\right| \downarrow \downarrow \ldots \downarrow\right\rangle$. If the rare region contains $N \sim L_{R R}^{d}$ spins, the lowest order that gives a nonzero contribution is $n=N$ because each $\sigma_{i}^{x}$ operator in the matrix element can only flip a single spin. We thus arrive at the important conclusion that the tunnel splitting (or energy gap) $\Delta$ must scale like $B^{N}$. In other words, the gap is exponentially small in the volume of the rare region,

$$
\Delta \sim B^{N} \sim \exp \left[-a L_{R R}^{d}\right]
$$

with $a \sim \ln (J / B)$.

We can calculate a rare-region density of states by combining the size dependence (75) of the energy gap with the probability (65) for finding a rare region. This gives

$$
\rho(\varepsilon)=\int d L_{R R} w\left(L_{R R}\right) \delta\left[\varepsilon-\Delta\left(L_{R R}\right)\right] \sim \varepsilon^{p / a-1}=\varepsilon^{\lambda-1}=\varepsilon^{d / z^{\prime}-1} .
$$


We thus find the (quantum) Griffiths phase to be gapless, featuring a power-law density of states. The exponent characterizing the singularity changes continuously throughout the Griffiths phase. It is often parameterized in terms of the Griffiths exponent $\lambda=p / a$ or the dynamical exponent $z^{\prime}=a d / p \cdot{ }^{30}$ In contrast to the classical case where the Griffiths singularities are weak essential singularities, the (quantum) Griffiths singularity in the density of states of the diluted transverse-field Ising model is a much stronger power-law singularity. If $z^{\prime}>d$, the density of states even diverges for $\varepsilon \rightarrow 0$.

In the ferromagnetic Griffiths phase, we can use the rare region probability (69) instead of (65). The resulting density of states is still gapless and singular but takes the form

$$
\rho(\varepsilon) \sim \exp \left\{-\tilde{c}\left[\ln \left(\varepsilon_{0} / \varepsilon\right)\right]^{1-1 / d}\right\}
$$

rather than a power law. Here, $\varepsilon_{0}$ represents a microscopic energy scale, and the constant $\tilde{c}=c / a^{1-1 / d}$ changes continuously throughout the ferromagnetic Griffiths phase.

\section{Observables in the quantum Griffiths phase}

Thermodynamic observables in the quantum Griffiths phase are easily calculated with the help of the rare region densities of state (76) or (77). Rare regions (locally ordered clusters) having gaps $\varepsilon<T$ are essentially free. This means both low-lying states are accessible. In contrast, clusters having gaps $\varepsilon>T$ are in their quantum ground state. The total number $n(T)$ of free clusters in the paramagnetic Griffiths phase can be obtained by integrating the density of states (76) up to energy $T$,

$$
n(T)=\int_{0}^{T} d \varepsilon \rho(\varepsilon) \sim T^{d / z^{\prime}}
$$

To determine the rare region entropy, we note that each free cluster contributes $k_{B} \ln (2)$ while the clusters having $\varepsilon>T$ do not contribute as they are frozen in their quantum ground state. We thus find

$$
S_{R R}(T)=k_{B} \ln (2) n(T) \sim T^{d / z^{\prime}}
$$

The rare region contribution to the specific heat can be calculated simply by taking the appropriate derivative

$$
C_{R R}(T)=T\left(\partial S_{R R} / \partial T\right) \sim T^{d / z^{\prime}} .
$$

Each free cluster makes a Curie contribution $\mu^{2} / T$ to the uniform magnetic susceptibility. According to (75), the volume (and thus the moment $\mu$ ) of a rare region depends logarithmically on its energy gap. The typical moment $\mu(T)$ of a free cluster at temperature $T$ thus depends only logarithmically on $T$. This weak dependence can be neglected

\footnotetext{
${ }^{30}$ To see that $z^{\prime}$ plays the role of a dynamical exponent, consider the typical distance $r_{\text {typ }}$ between excitations with energies below $\varepsilon$. Using (76), it follows that $r_{t y p} \sim \varepsilon^{-1 / z^{\prime}}$ or, equivalently, $\varepsilon \sim r_{t y p}^{-z^{\prime}}$. Thus $z^{\prime}$ indeed governs the relation between the energy and length scales in the quantum Griffiths phase.
} 
if we are only interested in the leading power-law behavior. The rare region susceptibility thus reads

$$
\chi_{R R}(T)=n(T) \mu^{2} / T \sim T^{d / z^{\prime}-1} .
$$

To find the zero-temperature magnetization in a small longitudinal magnetic field $h$, we note that all clusters having $\varepsilon<\mu h$ are almost fully polarized while clusters having $\varepsilon>$ $\mu h$ have negligible magnetization. The magnetization-field curve can thus be estimated as

$$
m_{R R}(h)=\int_{0}^{\mu h} d \varepsilon \rho(\varepsilon) \mu \sim h^{d / z^{\prime}} .
$$

Here, we have again neglected the subleading logarithmic dependence of the moment $\mu$ on the energy $\varepsilon$.

Many more observables can be worked out along the same lines including the nonlinear susceptibility $\chi^{(3)}(T) \sim T^{d / z^{\prime}-3}$, the zero-temperature dynamic susceptibility $\operatorname{Im} \chi(\omega) \sim \omega^{d / z^{\prime}-1}$, and the NMR relaxation rate $1 / T_{1} \sim \omega^{d / z^{\prime}-2}$. All these power laws constitute the famous quantum Griffiths singularities first discovered ${ }^{31}$ in the 1990's $[64,65,66,67]$. Recent overviews can be found, for example, in the review articles Refs. $[1,2]$. We emphasize that the power-law quantum Griffiths singularities are much stronger than the classical Griffiths singularities discussed in Sec. 4.2. The magnetic susceptibility (81) even diverges (when $z^{\prime}>d$ ) while the system is still in the paramagnetic phase.

It is instructive the compare the quantum Griffiths singularities (79) - (81) with the off-critical behavior (59) - (61) of the random transverse-field Ising chain calculated within the strong-disorder renormalization group. If we insert the correct dimensionality, $d=1$, the two sets of results agree. We thus conclude that the unusual off-critical behavior of the random-transverse-field Ising chain is a manifestation of the rare region physics discussed in the present section.

So far, we have focused on observables in the paramagnetic Griffiths phase. By using the density of states (77) rather than (76), one can also derive the quantum Griffiths singularities on the ferromagnetic side of the transition.

\subsection{Classification of Griffiths (rare region) effects}

In the last two subsections, we have compared the Griffiths singularities occurring at the thermal phase transition in a classical diluted Ising model with those occurring at the quantum phase transition in the diluted transverse-field Ising model. We have found the quantum Griffiths singularities to be much stronger as they are characterized by power laws rather than weak essential singularities. In recent years, rare regions have been studied in many other disordered classical, quantum, and nonequilibrium systems. The

\footnotetext{
31 Some of the unusual behavior was already identified by McCoy and Wu [61, 62, 63] in a classical Ising model with line defects. The transverse-field Ising chain maps onto this model under the quantum-toclassical mapping. For this reason, quantum Griffiths singularities are sometimes called Griffiths-McCoy singularities.
} 
qualitative features of the resulting Griffiths phenomena can be classified according to the effective dimensionality of the rare regions as was first suggested in Ref. [68] and further developed in Ref. [1].

To understand this classification, let us return to our simple estimate of the rare region susceptibility in the Griffiths phase. In general terms, it can be written as

$$
\chi_{R R} \approx \int d L_{R R} w\left(L_{R R}\right) \chi_{i}\left(L_{R R}\right)
$$

where $w\left(L_{R R}\right)$ is the probability for finding an individual rare region of size $L_{R R}$ and $\chi_{i}\left(L_{R R}\right)$ is its contribution to the susceptibility. The basic idea is to compare the decrease of the probability $w\left(L_{R R}\right)$ with the increase of $\chi_{i}\left(L_{R R}\right)$ as $L_{R R}$ is increased.

The functional form of $w\left(L_{R R}\right)$ is governed by combinatorics, it always decays exponentially with $L_{R R}$. More specifically, $w\left(L_{R R}\right) \sim \exp \left(-\tilde{p} L_{R R}^{d_{r}}\right)$ where $\tilde{p}$ is a nonuniversal constant and $d_{r}$ is the number of dimensions in which there is randomness. $d_{r}=d$ for point defects and other kinds of uncorrelated or short-range correlated randomness, but for extended (line or plane) defects $d_{r}<d$. The imaginary time dimension is never included in $d_{r}$ because quenched randomness is time-independent.

Depending on the behavior of $\chi_{i}\left(L_{R R}\right)$, three qualitatively different classes can be distinguished:

(A) $\chi_{i}\left(L_{R R}\right)$ increases slower than exponential with $L_{R R}$. Therefore, the increase in $\chi_{i}\left(L_{R R}\right)$ cannot overcome the exponential decay of the probability. The contribution of large rare regions to the susceptibility integral (83) is thus exponentially small. This case is realized, for instance, in the classical Ising model of Sec. 4.2 where each rare region makes a Curie contribution $\mu^{2}\left(L_{R R}\right) / T \sim L_{R R}^{2 d}$ to $\chi$.

(B) $\chi_{i}\left(L_{R R}\right)$ increases at least exponentially with $L_{R R}$ (but remains finite for any finite $\left.L_{R R}\right)$. In this case, the estimate (83) diverges which means that the susceptibility is dominated by the largest rare regions. The quantum Griffiths singularities of the transverse-field Ising model of Sec. 4.3 fall into this class because, according to (75), the zero-temperature susceptibility of a single rare region behaves as $1 / \Delta \sim$ $\exp \left(a L_{R R}^{d}\right)$.

(C) In this class, $\chi_{i}\left(L_{R R}\right)$ increases and diverges at some finite $L_{R R}$. This means that the cluster undergoes a true phase transition independently of the bulk system. As different rare regions generally undergo this transition at different values of the control parameter, this implies that the global phase transition is smeared. We will discuss this exotic possibility in Sec. 5.

Which of the three classes a system falls into is determined by the relation between the effective dimensionality $d_{R R}$ of the rare regions and the lower critical dimension $d_{c}^{-}$ of the problem. In the case of a quantum phase transition, the imaginary time dimension needs to be included in $d_{R R}$. If $d_{R R}>d_{c}^{-}$, the rare region can undergo a phase transition by itself, independently of the bulk. The system is therefore in class $C$. In the case $d_{R R}<d_{c}^{-}$, an isolated rare region cannot undergo the transition independently, and its contributions to observables grow like powers of $L_{R R}$. This transition is therefore in class A. In the marginal case when the rare regions are right at the lower critical dimension, $d_{R R}=d_{c}^{-}$, they still cannot undergo a transition by themselves, but they "almost can." 
TABLE 3. Classification of rare region effects at classical, quantum, and non-equilibrium phase transitions (after Ref. [68]). The first example in each case is a classical transition, the second one is a quantum phase transition, and the third one, if any, is a nonequilibrium transition. A detailed discussion of the classification and these examples is given in the reviews, Refs. [1, 2].

\begin{tabular}{ccccc}
\hline Class & $\begin{array}{c}\text { Rare region } \\
\text { dimension }\end{array}$ & $\begin{array}{c}\text { Griffiths } \\
\text { singularities }\end{array}$ & $\begin{array}{c}\text { Global phase } \\
\text { transition }\end{array}$ & $\begin{array}{c}\text { Examples } \\
\text { classical PT, QPT, non-eq. PT }\end{array}$ \\
\hline $\mathrm{A}$ & $d_{R R}<d_{c}^{-}$ & weak exponential & conventional & $\begin{array}{c}\text { 3D dilute Ising model [69] } \\
\text { dilute bilayer Heisenberg model [70] }\end{array}$ \\
B & $d_{R R}=d_{c}^{-}$ & strong power-law & $\begin{array}{c}\text { infinite } \\
\text { randomness }\end{array}$ & $\begin{array}{c}\text { Ising model with linear defects [61] } \\
\text { random transv.-field Ising model [44] } \\
\text { disordered directed percolation [71] }\end{array}$ \\
& & & & transition \\
C & $d_{R R}>d_{c}^{-}$ & $\begin{array}{c}\text { RRs frozen } \\
\text { (undergo PT) }\end{array}$ & $\begin{array}{c}\text { Ising model with planar defects [72] } \\
\text { itinerant quantum Ising magnet [73] } \\
\text { DP with extended defects [74] }\end{array}$ \\
\hline
\end{tabular}

Therefore, the rare region susceptibility (and other observables) increases exponentially with its volume, putting the system into class B.

The result of these ideas is the classification shown in Table 3. It is expected to hold for transitions between conventional phases in the presence of random mass disorder and short-range interactions (such that interactions between the rare regions can be neglected).

\section{SMEARED PHASE TRANSITIONS}

In all examples so far, the phase transition (if any) remained sharp in the presence of disorder. This means that a nonzero order parameter appears via a collective effect of the entire system; and this onset is associated with a singularity in the free energy. The reason for the transition remaining sharp is that a finite-size region usually does not undergo a true phase transition by itself.

However, the arguments in Sec. 4.4 suggest, that the global phase transition would be smeared rather than sharp if rare regions could develop true static order independently of the bulk system. In this case, the order parameter (e.g., the magnetization) would develop gradually when the external control parameter is varied because one spatial region after the other would undergo the phase transition.

How can an individual rare region undergo a true phase transition? One of the basic results of statistical physics is that the partition function of a finite-size system is analytic, and singularities develop only in the thermodynamic limit. This implies that either (i) the rare regions themselves must be infinitely large or (ii) they must at least be coupled to infinite baths. The first case is realized, for example, in a three-dimensional classical Ising model with plane defects [72]. It will be discussed in Sec. 5.1. The second mechanism can be found in dissipative quantum magnets [73] and will be analyzed in Sec. 5.2. 


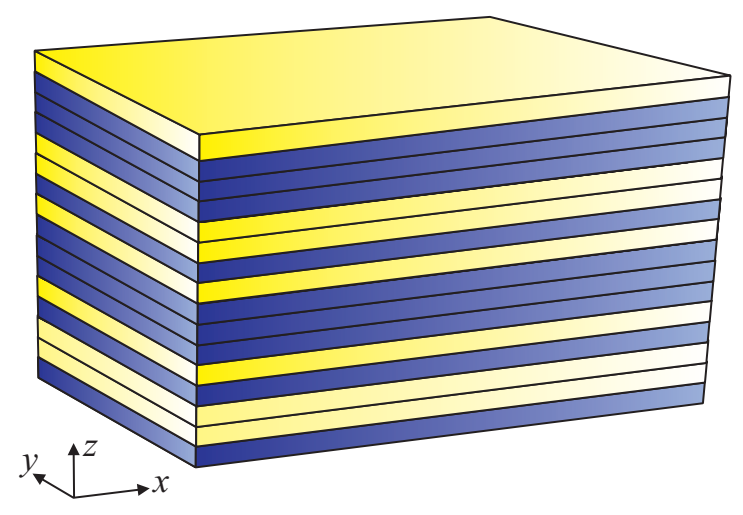

FIGURE 17. Schematic of the layered magnet: layers of two different ferromagnetic materials are arranged in a random sequence.

\subsection{Smearing of a classical phase transition}

\section{Randomly layered classical Ising model}

Imagine a material composed of a random sequence of layers of two different ferromagnetic materials, as sketched in Fig. 17. Nowadays, such structures can be created using modern nano-technology; magnetic multilayers with systematic variations of the critical temperature from layer to layer have already been produced [75].

The randomly layered magnet can be modeled by a three-dimensional classical Ising model

$$
H=-\sum_{\mathbf{r}} J_{z}^{\|}\left(S_{\mathbf{r}} S_{\mathbf{r}+\hat{\mathbf{x}}}+S_{\mathbf{r}} S_{\mathbf{r}+\hat{\mathbf{y}}}\right)-\sum_{\mathbf{r}} J_{z}^{\perp} S_{\mathbf{r}} S_{\mathbf{r}+\hat{\mathbf{z}}}
$$

where $S_{\mathbf{r}}$ is the spin at site $\mathbf{r}$, and $\hat{\mathbf{x}}, \hat{\mathbf{y}}, \hat{\mathbf{z}}$ are the unit vectors in the coordinate directions. The interactions $J_{z}^{\|}$within the layers and $J_{z}^{\perp}$ between the layers are random but depend on the $z$ coordinate only. For definiteness, let us assume all $J_{z}^{\perp}$ are identical to $J$ while the $J_{z}^{\|}$are drawn from a binary probability distribution

$$
J_{i}^{\|}=\left\{\begin{array}{cc}
J & (\text { with probability } 1-p) \\
a J & (\text { with probability } p)
\end{array} .\right.
$$

Here, $0<a \leq 1$ parameterizes the values of the weak interactions. $p$ is the probability for a weak layer while $1-p$ is the probability for a strong layer.

In the clean limit, $p=0$, the Hamiltonian (84) is identical to the usual threedimensional Ising model. It undergoes a sharp continuous phase transition at a critical temperature $T_{c}^{0} \approx 4.511 \mathrm{~J}$. For nonzero $p$, the overall tendency towards ferromagnetism is weakened. However, a large sample contains rare regions consisting of thick slabs of consecutive strong layers. In contrast to the rare regions considered in the earlier sections of this article, these slabs are infinite in the $x$ and $y$ directions. Each such region is 

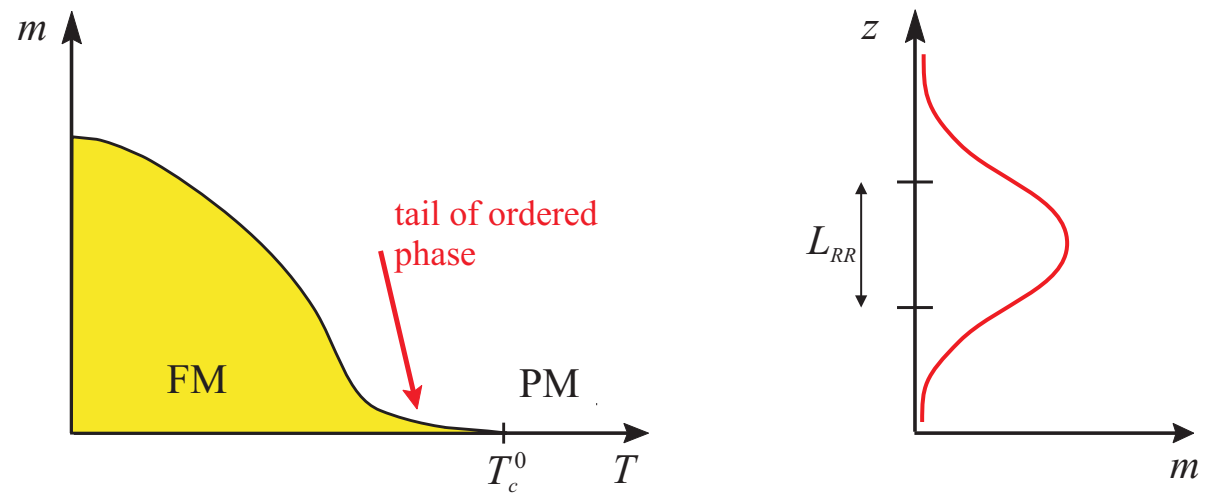

FIGURE 18. Left: Schematic behavior of the magnetization $m$ as a function of the temperature $T$ in the randomly layered Ising model (84). As the temperature is lowered below the clean critical temperature $T_{c}^{0}$, the magnetization increases gradually because one slab after the other undergoes the phase transition. The global transition is thus smeared. Right: Magnetization profile $m(z)$ of a single ferromagnetic rare region embedded in a paramagnetic bulk.

thus equivalent to a quasi two-dimensional Ising model. As the two-dimensional Ising model is known to have a ferromagnetic phase, each rare region in our layered magnet can undergo the phase transition independently of the bulk system.

The thickest slabs (rare regions) will undergo this transition very close to the threedimensional bulk critical temperature $T_{c}^{0}$. Thinner slabs order at lower temperatures. The ferromagnetic slabs are weakly coupled via fluctuations of the paramagnetic bulk, their magnetizations thus align. As a result, the global magnetization develops gradually when the temperature is lowered below $T_{c}^{0}$, as is sketched in Fig. 18. This means that the global phase transition is smeared over a range of temperatures below $T_{c}^{0}$. We emphasize that even though there is a singular onset of a nonzero magnetization at $T_{c}^{0}$, this point is not a critical point. Importantly, the initial onset of the global magnetization is not via a collective effect of the entire system but rather due to the phase transition of one, particularly thick "strong" slab. This also implies that the correlation length in $z$ direction remains finite for all $T$. The resulting magnetization in the tail of the smeared transition is extremely inhomogeneous in space.

\section{Optimal fluctuation theory}

We now use optimal fluctuation theory to determine the behavior in the tail of the smeared transition [72]. The approach is similar to the calculation by Lifshitz and others $[76,77,78]$ of the electronic density of states in the band tails of doped semiconductors.

As pointed out above, a thick slab of $L_{R R}$ strong layers (i.e., layers with $J^{\|}=J$ ) undergoes a ferromagnetic phase transition at some temperature $T_{c}\left(L_{R R}\right)$ below the clean three-dimensional bulk critical temperature $T_{c}^{0}$. We can use the LGW free energy (6) to determine how $T_{c}\left(L_{R R}\right)$ depends on the slab thickness $L_{R R}$. If we assume that the slab has undergone the transition and is embedded in a nonmagnetic bulk, its local magnetization 
profile $m(z)$ must roughly look like the right panel of Fig. 18. The (Gaussian part of the) free energy density due to this rare region can be estimated as $\Delta f_{R R} \sim t m^{2}+(\nabla m)^{2} \approx$ $t m^{2}+m^{2} / L_{R R}^{2}$. In mean-field theory, the transition of the slab occurs when the coefficient of $m^{2}$ vanishes, yielding $t_{c}\left(L_{R R}\right) \sim T_{c}\left(L_{R R}\right)-T_{c}^{0} \sim-1 / L_{R R}^{2}$. The mean-field estimate can be refined using finite-size scaling [25] at the three-dimensional bulk critical point. This results in

$$
T_{c}^{0}-T_{c}\left(L_{R R}\right) \sim L_{R R}^{-\phi}
$$

where $\phi$ is the finite-size scaling shift exponent of the three-dimensional bulk critical point. It takes the value $\phi=1 / v$ because the three-dimensional Ising model is below its upper critical dimension of $d_{c}^{+}=4$.

At a given temperature $T<T_{c}^{0}$, all slabs thicker than a critical thickness $L_{c}(T) \sim$ $\left(T_{c}^{0}-T\right)^{-v}$ are in the ferromagnetic phase while the rest of the system is still in the paramagnetic phase. Close to $T_{c}^{0}$, the density of ferromagnetic slabs is very small, thus they can be considered independent. The total magnetization in the tail of the smeared transition is therefore simply the sum over all ferromagnetic slabs,

$$
m \sim \int_{L_{c}}^{\infty} d L_{R R} w\left(L_{R R}\right) m\left(L_{R R}\right) .
$$

Here $m\left(L_{R R}\right)$ is the magnetization of a single ferromagnetic slab, and $w\left(L_{R R}\right)$ is the probability of finding $L_{R R}$ consecutive strong slabs. This probability is given by the previous result (65), but with the dimension $d$ replaced by $d_{r}=1$ because our layered Ising model has randomness in one dimension (the $z$ direction) only. Thus, $w\left(L_{R R}\right) \sim$ $\exp \left(-\tilde{p} L_{R R}\right)$ with $\tilde{p}=-\ln (1-p)$. While the probability $w\left(L_{R R}\right)$ depends exponentially on the slab thickness, the dependence of the slab magnetization $m\left(L_{R R}\right)$ on $L_{R R}$ is at best a power law. It thus provides a subleading correction to the integral (87). To exponential accuracy, we find

$$
m \sim \exp \left(-\tilde{p} L_{c}\right) \sim \exp \left[-A\left(T_{c}^{0}-T\right)^{-v}\right]
$$

with $A$ being a nonuniversal constant.

The magnetization thus decays exponentially in the tail of the smeared transition and vanishes with an essential singularity at the clean bulk critical temperature $T_{c}^{0}$. It is important to keep in mind that this total (average) magnetization stems from a very inhomogeneous spatial distribution. This can be seen in the results of large-scale Monte Carlo simulations [79] of the layered Ising model that are shown in Fig. 19. Other observables can be worked out in a similar fashion [72, 79].

We emphasize that the functional form (88) of the magnetization as well as those of other observables in the tail of the smeared transition are not universal. In contrast to the behavior at critical points, these functional forms do depend on the details of the disorder distributions and on how the phase transition is tuned. If one uses, for example, a Gaussian interaction distribution instead of the binary distribution (85), the magnetization tail stretches all the way to $T=\infty$ [72]. Moreover, if one tunes the phase transition by varying the concentration $p$ of the weak interactions at fixed $T$, the 

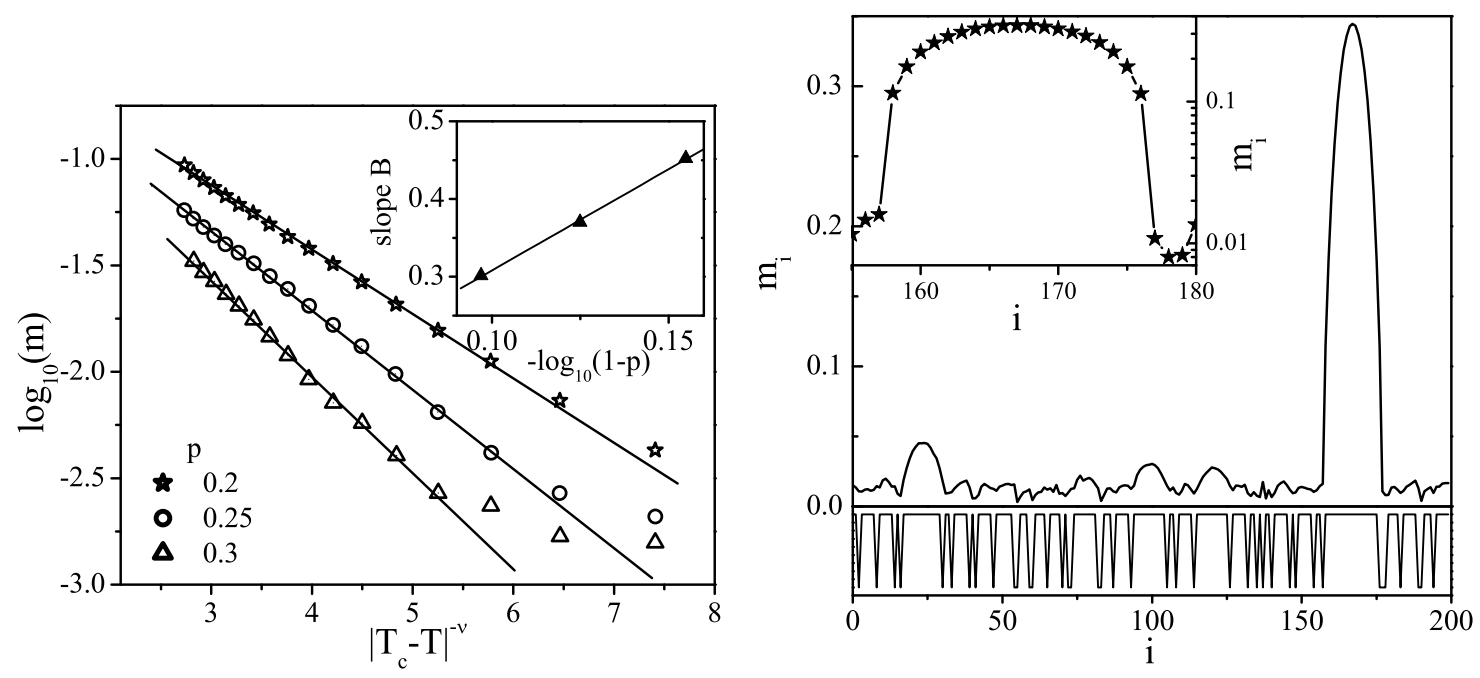

FIGURE 19. Monte-Carlo simulations of a randomly layered Ising model. Left: Total magnetization $m$ in the tail of the smeared transition for several values of the weak bond probability $p$. Right: Local magnetization $m_{i}$ of layer $i$ for one disorder realization at $T=4.425$ very close to $T_{c}^{0}=4.511$. Only one slab has developed ferromagnetic order, and two or three more seem to be at the verge of ordering while the bulk magnetization vanishes within the Monte-Carlo noise. The interaction $J_{i}$ at the corresponding position is indicated in the lower panel (after Ref. [79]).

magnetization tail behaves exponentially at intermediate $p$ but vanishes as a power-law for $p \rightarrow 1[80,81]{ }^{32}$

\subsection{Smearing of a quantum phase transition}

\section{Dissipative transverse-field Ising chain}

We now discuss the second possible route to smeared phase transitions in which individual rare regions undergo their transitions independently because they are coupled to infinite (dissipative) baths.

We start from the random transverse-field Ising chain (27) that we discussed in detail in Sec. 3. Imagine that we now couple the $z$ component of each spin to an independent (infinite) bath of quantum harmonic oscillators. The resulting Hamiltonian, the dissipative random transverse-field Ising chain, can be written as $H=H_{I}+H_{B}+H_{C}$. The Ising model part,

$$
H_{I}=-\sum_{i} J_{i} \sigma_{i}^{z} \sigma_{i+1}^{z}-\sum_{i} B_{i} \sigma_{i}^{x}
$$

32 See also the article by Nozadze et al. in this proceedings volume. 
is identical to (27), the bath Hamiltonian takes the form

$$
H_{B}=\sum_{k, i} \hbar \omega_{k, i}\left(a_{k, i}^{\dagger} a_{k, i}+1 / 2\right)
$$

and the coupling between the spins and the dissipative baths is given by

$$
H_{C}=\sum_{i} \sigma_{i}^{z} \sum_{k} \lambda_{k, i}\left(a_{k, i}^{\dagger}+a_{k, i}\right)
$$

Here, $a_{k, i}^{\dagger}$ and $a_{k, i}$ are the creation and destruction operators of oscillator $k$ coupled to spin $i$, and $\omega_{k, i}$ is its frequency. The $\lambda_{k, i}$ parameterize the strength of the coupling between spins and oscillator baths. The character of the baths depends crucially on the low-frequency behavior of their spectral densities

$$
\mathscr{E}_{i}(\omega)=\pi \sum_{k} \lambda_{k, i}^{2} \delta\left(\omega-\omega_{k, i}\right)
$$

The most important case is, arguably, Ohmic ${ }^{33}$ dissipation for which the spectral density vanishes linearly with $\omega$ in the low frequency limit, $\mathscr{E}_{i}=(\pi / 2) \alpha_{i} \omega$ for frequencies below a cutoff $\omega_{c}$.

The dissipative random transverse-field Ising chain can be attacked by a generalization of the strong-disorder renormalization group technique of Sec. 3. This was first done by implementing the recursion relations (which now also include the renormalizations of the oscillators) numerically $[82,83]$. Later, a complete analytical solution was found $[84,85]$. The renormalization group analysis is mathematically rather involved and thus beyond the scope of these introductory lectures. Here, we instead develop a heuristic picture of the rare region properties in the presence of Ohmic dissipation.

Consider again a rare locally ferromagnetic region of length $L_{R R}$ embedded in a paramagnetic bulk. (This could be a region in which a number of consecutive interactions $J_{i}$ are atypically strong or in which a few consecutive transverse fields are atypically weak.) As discussed in Sec. 4.3, the low-energy spectrum of such a region is identical to that of a quantum two-level system. When coupled to Ohmic dissipative baths, each rare region is thus equivalent to a dissipative two-level system which is a famous, paradigmatic model in quantum physics $[86,87,88]$.

Importantly, the dissipative two-level system undergoes a (zero-temperature) quantum phase transition from a fluctuating ground state (a symmetric superposition of $|\uparrow\rangle$ and $|\downarrow\rangle$ ) to a localized ground state (which prefers either $|\uparrow\rangle$ or $|\downarrow\rangle$ ) as the dissipation strength $\alpha$ is increased beyond a critical value $\alpha_{c}$. In our case, the effective dissipation strength of a rare region is simply the sum over the $\alpha_{i}$ of all baths that couple to the rare region, it is thus proportional to its size, $\alpha_{\text {eff }}=\sum_{i} \alpha_{i} \sim L_{R R}$. Consequently, sufficiently large rare regions have effective dissipation strengths $\alpha_{\text {eff }}>\alpha_{c}$ even if the initial (bare) dissipation strengths $\alpha_{i}$ are weak. Large rare regions therefore localize, i.e., they undergo

33 An Ohmic dissipative bath leads to a friction force that is proportional to the velocity and thus to Ohm's law when applied to the motion of charge carriers in a conductor. 


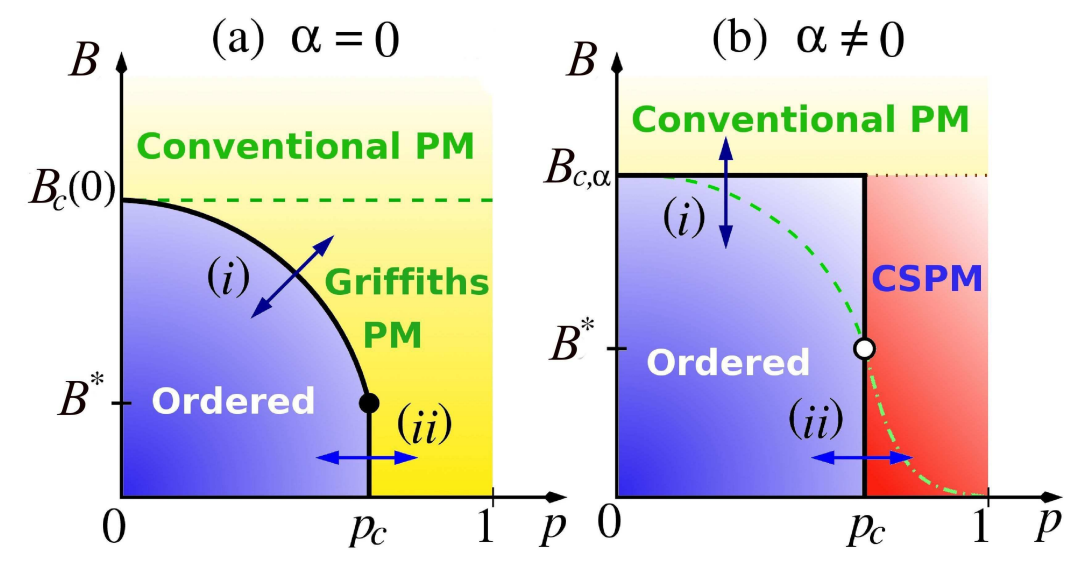

FIGURE 20. Schematic zero-temperature phase diagrams of diluted transverse-field Ising magnets without (a) and with (b) Ohmic dissipation. In the classical super-paramagnetic phase (CSPM), large rare regions are frozen and act as classical superspins. The dashed line in (b) marks the crossover between homogeneous and inhomogeneous order in the smeared transition scenario (after Ref. [89]).

the ferromagnetic quantum phase transition independently of the bulk system. This implies that the global quantum phase transition is smeared. This conclusion agrees with the explicit strong-disorder renormalization group calculations [82, 83, 84, 85]. The behavior of observables can be determined either by means of the renormalization group or by employing an optimal fluctuation approach similar to that of Sec. 5.1.

\section{Higher dimensions}

Similar phenomena also arise if we couple Ohmic dissipative oscillator baths to the two-dimensional or three-dimensional site-diluted transverse-field Ising model (74). This problem was studied in Ref. [89]. Sufficiently large percolation clusters are on the localized side of the dissipative two-level system quantum phase transition because their effective dissipation strength $\alpha_{\text {eff }}$ is larger than $\alpha_{c}$. This implies that their quantum dynamics is frozen.

Consequently, the phase diagram of the model changes qualitatively compared to the dissipationless case, as is demonstrated in Fig. 20. For dilutions $p<p_{c}$, the lattice remains connected. The large ferromagnetic rare regions thus align with each other. This leads to a smeared phase transition as $B$ is varied at fixed $p$ [transition (i) in Fig. 20(b)]. The tail of the ferromagnetic phase stretches all the way to the clean critical field $B_{c, \alpha}$. For dilutions $p>p_{c}$, in contrast, the lattice consists of disconnected clusters. Long-range ferromagnetic order is thus impossible. The large ferromagnetic rare regions therefore act as independent superspins. Because their quantum dynamics is frozen, their behavior is purely classical.

We conclude that the Ohmic dissipation completely destroys the quantum Griffiths phase. For $p<p_{c}$, it is replaced by the tail of the smeared ferromagnetic phase transition, and for $p>p_{c}$, it is supplanted by a classical super-paramagnetic phase. In contrast to the 
field-driven transition (i), the percolation transition (ii) between the ferromagnetic phase and the classical super-paramagnet remains sharp because it is driven by the geometry of the underlying lattice.

We emphasize that all these conclusions hold at zero temperature. At nonzero temperatures, the static order of the rare regions is destroyed, and Griffiths singularities may be present in a transient temperature and energy range. We will come back to this point in the next subsection.

\section{MAGNETIC QUANTUM PHASE TRANSITIONS IN METALS}

\subsection{Landau-Ginzburg-Wilson theory}

A particularly important application of the ideas developed in the last sections are magnetic quantum phase transitions in metallic materials. The standard theory of quantum phase transitions in (clean) Fermi liquids is the Hertz-Millis theory [90, 91]. It can be derived by starting from a microscopic Hamiltonian of interacting electrons and integrating out all fermionic degrees of freedom; only the order parameter (magnetization) fluctuations $m(\mathbf{x}, \tau)$ are kept in the partition function. The result is a quantum LGW free energy (or action) of the form

$$
\begin{aligned}
S[m(\mathbf{x}, \tau)]= & \int d^{d} x_{1} d \tau_{1} \int d^{d} x_{2} d \tau_{2} m\left(\mathbf{x}_{\mathbf{1}}, \tau_{1}\right) \Gamma\left(\mathbf{x}_{\mathbf{1}}, \tau_{1}, \mathbf{x}_{\mathbf{2}}, \tau_{2}\right) m\left(\mathbf{x}_{\mathbf{1}}, \tau_{1}\right) \\
& +u \int d^{d} x d \tau m^{4}(\mathbf{x}, \tau)+O\left(m^{6}\right)
\end{aligned}
$$

The order-parameter $m$ is a scalar for easy-axis (Ising) magnets but a two-component or three-component vector for the cases of XY or Heisenberg symmetries, respectively. The two-point vertex $\Gamma\left(\mathbf{x}_{1}, \tau_{1}, \mathbf{x}_{2}, \tau_{2}\right)$ is given by its Fourier transform

$$
\Gamma\left(\mathbf{q}, \omega_{n}\right)=r+\mathbf{q}^{2}+\gamma(\mathbf{q})\left|\omega_{n}\right| .
$$

Here, $\mathbf{q}$ is the wave number of the order parameter fluctuations, and $\omega_{n}$ is a Matsubara frequency.

In contrast to the example LGW theory (13), the dynamic term $\gamma(\mathbf{q})\left|\omega_{n}\right|$ is nonanalytic in the frequency. This is an example of the nonanalyticities that generally occur whenever gapless (soft) modes are integrated out in the derivation of the LGW theory [28]. Physically, this term accounts for the so-called Landau damping of the order parameter fluctuations by the excitation of fermionic particle-hole pairs. The wave number dependence of the prefactor $\gamma(\mathbf{q})$ depends on the type of quantum phase transition. For a ferromagnetic transition $\gamma(\mathbf{q})$ behaves as $1 /|\mathbf{q}|$ for $\mathbf{q} \rightarrow 0$, reflecting the order parameter conservation in a metallic ferromagnet. For a generic antiferromagnetic transition (as well as the pair-breaking superconducting transition), $\gamma(\mathbf{q})=\gamma_{0}=$ const for $\mathbf{q} \rightarrow 0 .{ }^{34}$

\footnotetext{
34 The Hertz-Millis theory does not apply to all quantum phase transitions in metals, and at least two scenarios leading to its breakdown have been identified. (i) The coupling of the order parameter to other 
To find the LGW theory for the case of a disordered metal, one can repeat the derivation starting from a model of electrons in the presence a random potential or some other kind of disorder. Hertz [90] found that the structure of the action remains essentially unchanged. ${ }^{35}$ However, the distance from criticality becomes a random function of spatial position, $r \rightarrow r+\delta r(\mathbf{x})$. This means, the action contains random-mass disorder.

It is instructive to compare the Hertz-Millis action to the LGW theory of the dissipative random-transverse-field Ising model considered in Sec. 5.2. This LGW theory, which can be obtained by integrating out the Ohmic oscillator baths in the partition function, turns out to be identical to the Hertz-Millis action with a scalar order parameter and $\gamma(\mathbf{q})=$ const. The nonanalytic frequency dependence $\left|\omega_{n}\right|$ is a reflection of the Ohmic character of the dissipative baths.

In following, we discuss the properties of quantum phase transitions occurring in the Hertz-Millis LGW theory with random mass disorder. We first focus on the case $\gamma(\mathbf{q})=$ const, valid for antiferromagnetic transitions (and pair-breaking superconducting transitions). The more complicated ferromagnetic case, $\gamma(\mathbf{q}) \sim 1 / \mathbf{q}^{2}$ will be addressed later.

\section{Ising symmetry: smeared quantum phase transition}

The character of the transition depends crucially on the order-parameter symmetry. For Ising symmetry, each locally ordered rare region can undergo the quantum phase transition independently of the bulk system. This follows from the equivalence pointed out above of the Hertz-Millis theory and the dissipative transverse-field Ising model. The same conclusion can also be drawn directly from the LGW theory $(93,94)$. After quantum-to-classical mapping, a rare region is equivalent to a quasi one-dimensional rod with finite size in the space dimensions, but infinitely long in the imaginary time direction (see left panel of Fig. 21). As the nonanalytic $\left|\omega_{n}\right|$ frequency dependence corresponds to a long-range $\left|\tau_{1}-\tau_{2}\right|^{-2}$ interaction in the imaginary time direction, each individual rare region is equivalent to a one-dimensional Ising model with a $1 / \tau^{2}$ interaction. This model in known to have a phase transition $[94,95]$ implying that each rod can order independently. This result was also reached by Millis, Morr, and Schmalian via an instanton analysis of the Hertz-Millis action [96, 97]. The conclusion of all these considerations is that the global (zero-temperature) quantum phase transition is smeared [73]. Observables can be calculated, for instance, by adapting the optimal fluctuation theory of Sec. 5.1 .

As all experiments are performed at finite temperatures, it is important to ask: What happens to the smeared quantum phase transition as the temperature is raised? In the

(generic) soft modes present in the system can invalidate the entire expansion in powers of the order parameter [28]. (ii) Additional degrees of freedom other than the order parameter fluctuations become critical at the transition point. This mechanism seems to play a role at magnetic transitions in some heavyfermion compounds [92, 93].

35 In a ferromagnet, $\gamma(\mathbf{q})$ now behaves as $1 / \mathbf{q}^{2}$ because the electron motion is diffusive rather than ballistic. 

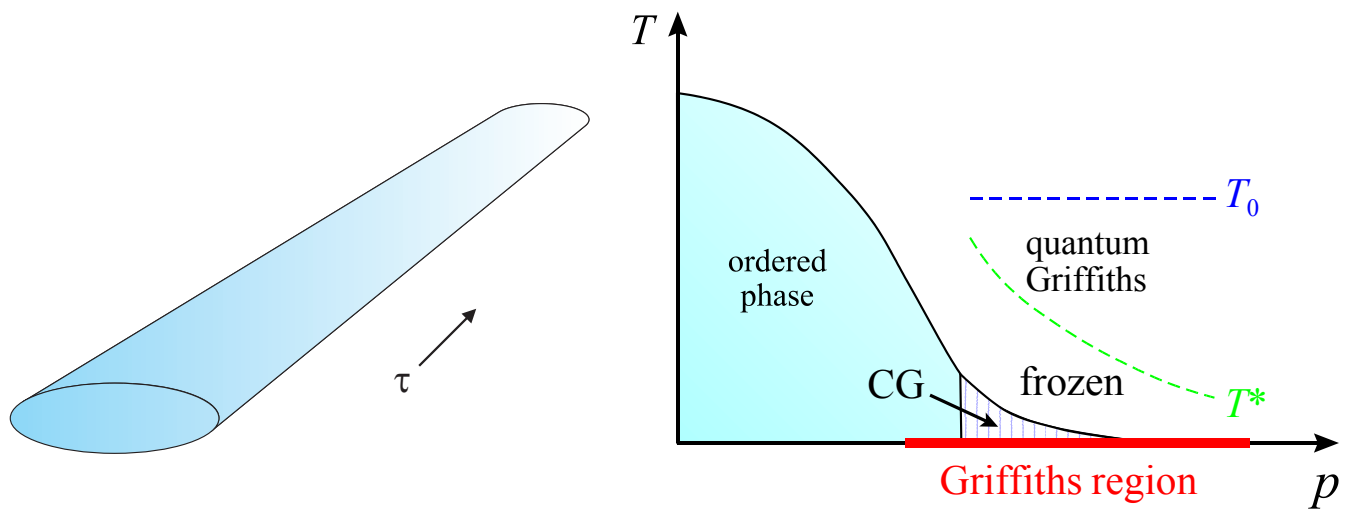

FIGURE 21. Left: A rare region in the LGW theory $(93,94)$ is finite in space but infinitely long in the imaginary time direction. Right: Schematic finite-temperature phase diagram near the smeared quantum phase transition. $p$ stands for the quantum tuning parameter, and $\mathrm{CG}$ denotes the cluster glass expected due to the RKKY interaction between the rare regions in a realistic metal. $T^{*}$ marks the crossover between fluctuating and frozen rare regions, and the behavior above the microscopic cutoff $T_{0}$ is nonuniversal.

tail of the smeared transition, the locally ordered rare regions are far apart and thus only weakly coupled. Their relative alignment is therefore destroyed at a very low temperature. This leads to an exponential dependence of the critical temperature on the quantum tuning parameter, as shown in the right panel of Fig. 21. Above the critical temperature, the rare regions act as independent classical moments.

The behavior at higher temperatures depends on the strength of the damping, i.e., on the strength (prefactor) of the leading $\left|\omega_{n}\right|$ frequency dependence compared to the regular (but subleading) $\omega_{n}^{2}$ term that is always present in the LGW expansion. If the damping is weak, it becomes important only below a crossover temperature $T^{*}$. Above this temperature, the quantum dynamics of the rare regions is effectively undamped, leading to the quantum Griffiths singularities of Sec. 4.3 in a wide temperature window [98, 99]. In contrast, if the damping is strong, this window becomes very narrow or completely unobservable. The actual strength of the damping in realistic metallic magnets has been discussed controversially in the literature.

As is the case for smeared classical transitions, the properties of smeared quantum phase transitions are nonuniversal. They depend on the details of the disorder distributions as well as on how the transition is tuned. The experimentally important case of tuning by chemical composition is addressed in Refs. [80, 81].

\section{Continuous symmetry: quantum Griffiths singularities}

In the case of continuous $O(N)$ order parameter symmetry (which includes XY and Heisenberg symmetry), the behavior of locally ordered rare regions is qualitatively different. After the quantum-to-classical mapping, each rare region now corresponds to a quasi one-dimensional $O(N)$ model with $1 / \tau^{2}$ interactions. These models are known not to have a phase transition, but they are exactly at their lower critical dimension $d_{c}^{-}$ $[100,101,102]$. For this reason, an isolated rare region cannot independently undergo 
the quantum phase transition. However, its characteristic energy depends exponentially on its volume. According to the classification of rare region effects discussed in Sec. 4.4, the problem is thus in class B.

This prediction was confirmed by an explicit analysis of the rare region effects in the LGW theory (93) with $O(N)$ order parameter symmetry [68]. This work established power-law quantum Griffiths singularities very similar to that of the transverse-field Ising model discussed in Sec. 4.3. ${ }^{36}$ Later, Hoyos et al. [104, 105] applied a strongdisorder renormalization group to this problem. Somewhat surprisingly, they found an infinite-randomness quantum critical point in the same universality class as the randomtransverse field Ising model.

This result points to an unusual kind of super-universality that is not fully understood. Normally, critical points are in the same universality class (i.e., they share the same exponent values) if the symmetries of the problems are the same. However, the random transverse-field Ising model has discrete Ising order parameter symmetry and undamped dynamics while the disordered $O(N)$ Hertz-Millis theory has a continuous symmetry order parameter and Ohmic damping. Nonetheless, the critical points are in the same universality class.

We emphasize that all these studies assume that the interactions between different rare regions are weak. Dobrosavljevic and Miranda [106] studied the effects of the longrange RKKY interaction between magnetic moments. It is not contained in the HertzMillis theory but does exist in realistic metals. They found that this interaction leads to a spin-glass like freezing of the rare regions at the lowest temperatures (well below the energy scale of the single rare-region effects) even in the continuous-symmetry case.

\subsection{Experiments}

Clear-cut experimental verifications of the strong-disorder effects discussed in these lectures were lacking for a long time. In the last five years, however, a number of promising experiments have appeared.

Guo et al. [107, 108, 109] studied the ferromagnetic quantum phase transition occurring in the magnetic semiconductor $\mathrm{Fe}_{1-x} \mathrm{Co}_{x} \mathrm{~S}_{2}$ at a concentration $x_{c}$ of about $0.007 \pm 0.002$. Close to the critical concentration, the authors found fluctuating magnetic moments with sizes significantly larger than the expected spin-1/2 moments of individual $\mathrm{Co}$ atoms in an $\mathrm{FeS}_{2}$ host. This suggests that locally ordered magnetic clusters are forming. The unusual transport, magnetic, and thermodynamic properties in this concentration range can be described in terms of the quantum Griffiths power laws listed in section 4.3 .

The $f$-electron Kondo lattice system $\mathrm{CePd}_{1-x} \mathrm{Rh}_{x}$ undergoes a ferromagnetic quantum phase transition as a function of the rhodium concentration $x$ [110]. CePd is a ferromagnet with a critical temperature of $T_{c}=6.6 \mathrm{~K}$ while $\mathrm{CeRh}$ has a non-magnetic ground state. The ferromagnetic phase develops a pronounced tail from about $x=0.7$ to 0.9 .

${ }^{36}$ Griffiths singularities appear not just in the thermodynamics but also in transport properties [103]. 


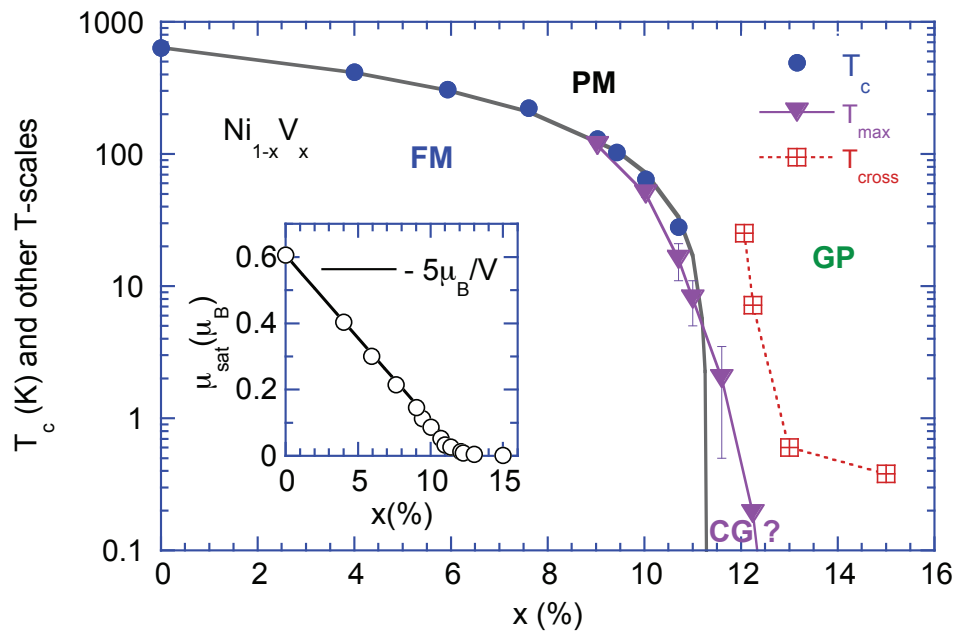

FIGURE 22. Temperature-concentration phase diagram of $\mathrm{Ni}_{1-x} \mathrm{~V}_{x}$ showing ferromagnetic (FM), paramagnetic (PM), quantum Griffiths (GP), and cluster glass (CG) phases. Inset: saturation magnetization $\mu_{\text {sat }}$ versus $x$ (after Ref. [112]).

Above this tail (at temperatures above $T_{c}$ ), the behavior of the magnetic susceptibility, the specific heat, and other observables is characterized by nonuniversal power laws in $T$ whose exponents vary systematically with $x$ [111]. Moreover, at the lowest temperatures, indications of a "cluster glass" are found. All these observations can be attributed to the scenario outlined in Sec. 6.1 (see Fig. 21).

One of the most convincing verifications of quantum Griffiths behavior in a metallic Heisenberg magnet can be found in the transition metal alloy $\mathrm{Ni}_{1-x} \mathrm{~V}_{x}$. Nickel is a ferromagnetic metal with a high Curie temperature of about $T_{c}=630 \mathrm{~K}$. Vanadium substitution quickly suppresses the ferromagnetic order, leading to the phase diagram shown in Fig. 22. Ubaid-Kassis et al. [112, 113] performed magnetization and a.c. susceptibility measurements of several samples located just on the paramagnetic side of the quantum phase transition (samples with vanadium concentrations $x$ between the critical concentration of about $11 \%$ and 15\%). The results are summarized in Fig. 23. The figure shows that the data (for $T>10 \mathrm{~K}$ and $H>3000 \mathrm{G}$ ) can be well described by nonuniversal power laws, as predicted in Sec. 4.3 for quantum Griffiths singularities. The Griffiths exponent $\lambda=d / z^{\prime}$ can be determined from fits of the data to (81) and (82). As expected in the quantum Griffiths scenario, the values extracted from the susceptibility and from the magnetization-field curve agree. Moreover, $\lambda$ vanishes (and $z^{\prime}$ diverges) at the critical point $x_{c} \approx 11.4 \%$, as predicted. We note in passing that the measurements show indications of a cluster glass at concentrations close to $x_{c}$ and very low temperatures (below about $1 \mathrm{~K}$ ), in agreement with the scenario of Ref. [106].

Our final example is the ferromagnetic quantum phase transition that occurs in $\mathrm{Sr}_{1-x} \mathrm{Ca}_{x} \mathrm{RuO}_{3}$ as a function of composition $x$. The group of Kézsmarki studied this transition using a highly sensitive magneto-optical technique [20]. The resulting phase diagram, which was already presented in Fig. 6, shows a pronounced tail of the ferromagnetic phase boundary. The magnetization-concentration curve features a similar 


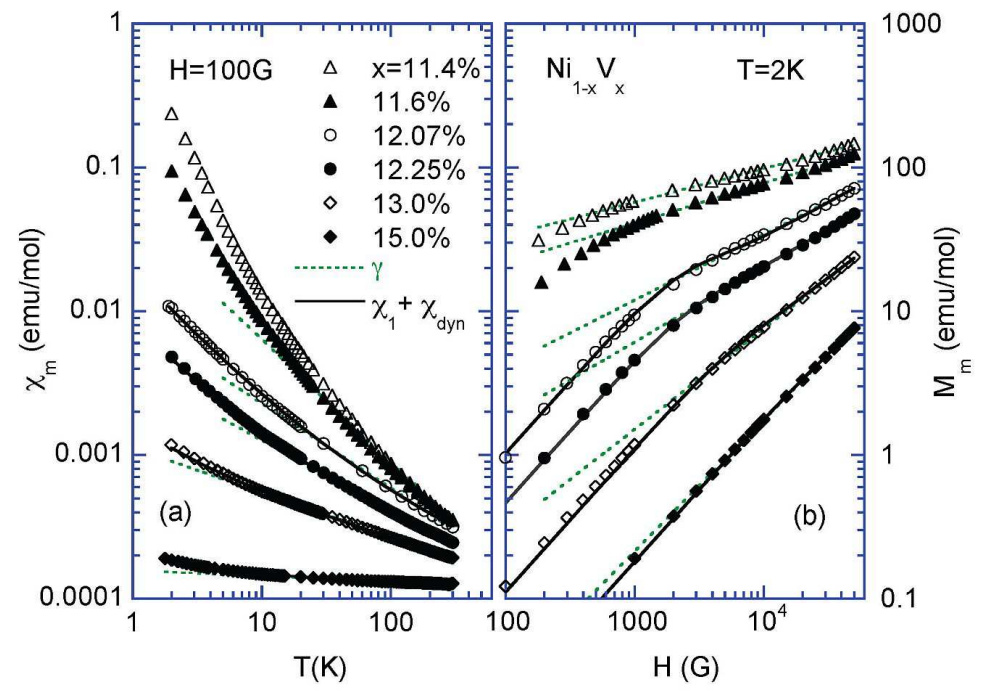

FIGURE 23. (a) Low-field susceptibility $\chi_{m}$ versus temperature $T$ for several $\mathrm{Ni}_{1-x} \mathrm{~V}_{x}$ samples with concentrations $x=11-15 \%$ and (b) low-temperature magnetization $M_{m}$ vs magnetic field $H$. Dotted lines indicate power-law fits for $T>10 \mathrm{~K}$ and $H>3000 \mathrm{G}$ in (a) and (b), respectively (after Ref. [112]).

tail. Both tails can be fitted well with the theory of composition-tuned smeared quantum phase transitions developed in Refs. [80, 81].

The attentive reader may have noticed that all the above examples involve ferromagnetic quantum phase transitions in metals while the theoretical analysis of Sec. 6.1 focused on the antiferromagnetic transition. This is not an accident. Somewhat ironically, all good experimental examples of quantum Griffiths physics appear to be found at ferromagnetic transitions while the theory of rare region effects in metals is better developed for antiferromagnetic transitions. The reason that quantum Griffiths singularities in ferromagnetic metals remained unexplored by theory for a long time is the nonlocal character $|\omega| / \mathbf{q}^{2}$ of the damping term which complicates the theoretical analysis. This problem was solved very recently in Ref. [114]. The resulting rare region density of states is not a pure power law but rather takes the form $\rho(\varepsilon) \sim(1 / \varepsilon) \exp \left[-\tilde{\lambda} \ln ^{3 / 5}(1 / \varepsilon)\right]$. The functional forms of observables are changed accordingly. It turns out that these ferromagnetic Griffiths singularities actually lead to improved fits [114] of the $\mathrm{Ni}_{1-x} \mathrm{~V}_{x}$ data shown in Fig. 23.

\section{CONCLUSIONS AND OUTLOOK}

These lectures notes have given an introduction into the effects of random disorder on phase transitions in quantum systems and into the exotic Griffiths phases occurring in the vicinity of such transitions.

We have seen that zero-temperature quantum phase transitions generically display stronger disorder effects than thermal (classical) phase transitions. The reason is that quenched disorder is perfectly correlated in the imaginary time direction which needs to be taken into account at a quantum phase transition. At zero temperature, the defects are 
effectively infinite in this direction, enhancing their effects.

This implies that similarly strong disorder effects should occur at thermal transitions in the presence of extended defects. This has indeed been observed not just in the famous McCoy-Wu model [61, 62, 63] or in the layered classical Ising model [79] discussed in Sec. 5.1, but also in randomly layered Heisenberg [115, 116] and XY [117] models. The latter problem is particularly interesting because it applies to the superfluid phase transition of ultracold bosons [118].

Two different classifications of disorder effects have emerged from our discussion. First, one can classify critical points according to the behavior of the average disorder strength in the limit of large length scales [119]. Three cases can be distinguished. (i) If the Harris criterion is fulfilled, the disorder strength goes to zero, and the critical behavior is identical to that of the clean system. The other two cases occur if the Harris criterion is violated. (ii) If the effective disorder strength remains nonzero and finite in the large-length scale limit, the clean critical behavior is unstable. The disordered critical point still features conventional power-law scaling, but with exponents that differ from the clean ones. (iii) If the effective disorder strength diverges, the resulting exotic infinite-randomness critical point features activated (exponential) dynamical scaling rather than the usual power-law scaling. Smeared phase transitions do not fit very well into this classification scheme because qualitatively new physics (namely, the freezing of the locally ordered rare regions) happens at a finite length scale, destroying the critical point.

The second classification $[68,1]$ focuses on the properties of the rare regions and was discussed in Sec. 4.4. Depending on the effective dimensionality $d_{R R}$ of the rare regions, the following classes can be distinguished. (A) The rare regions are below the lower critical dimension $d_{c}^{-}$of the problem. This leads to (exponentially) weak Griffiths singularities and critical points with conventional scaling. (B) If the rare regions are right at the lower critical dimension (but still cannot order independently) the Griffiths singularities are of power-law type and the critical point is an exotic infinite-randomness critical point. (C) If the rare regions are above the lower critical dimension, they can undergo the phase transition independently of the bulk system. The global transition is thus smeared. These two classifications are not independent of each other, as can be seen in Table 3. Together, they form the basis of our current understanding of quantum phase transitions in the presence of random-mass disorder.

The scenarios developed in these lectures apply to quantum phase transitions that can be described by Landau-Ginzburg-Wilson order parameter field theories with real actions (such that the quantum-to-classical mapping is valid). The effects of disorder on other, unconventional quantum phase transitions is less well understood. This includes transitions in Fermi liquids not described by the Hertz-Millis theory, Kondo lattice systems, quantum magnets in which the Berry phases are important, or other systems with topological excitations. Some such transitions have already been studied and revealed novel, exotic types of phase transitions. For example, Fernandes and Schmalian studied the quantum phase transition in a diluted Josephson junction array [120]. They found that a topological Berry phase in this problem renders the critical exponents complex, a striking deviation from the Landau paradigm.

A systematic investigation of disorder effects at unconventional (non-Landau) transitions remains a task for the future. 


\section{ACKNOWLEDGMENTS}

The author is grateful for the hospitality of the organizers of the XVIIth Training Course in the Physics of Strongly Correlated Systems, Dr. Avella and Prof. Mancini, as well as the local staff in Vietri sul Mare. It was a great pleasure to lecture at the course. Parts of the work reported here were supported by the National Science Foundation under Grant Nos. DMR-0339147, DMR-0906566, and DMR-1205803, as well as by Research Corporation.

\section{REFERENCES}

1. T. Vojta, J. Phys. A 39, R143 (2006).

2. T. Vojta, J. Low Temp. Phys. 161, 299 (2010).

3. F. Igloi, and C. Monthus, Phys. Rep. 412, 277 (2005).

4. N. Goldenfeld, Lectures on phase transitions and the renormalization group, Addison-Wesley, Reading, 1992.

5. S. Sachdev, Quantum phase transitions, Cambridge University Press, Cambridge, 1999.

6. $\quad$ P. Ehrenfest, Proc. Kon. Ned. Akad. Wet. (Amsterdam) 36, 153 (1933).

7. T. Andrews, Phil. Trans. R. Soc. 159, 575 (1869).

8. M. de Podesta, Critical opalescence (2013), http://www.physicsofmatter.com/.

9. $\quad$ R. Swendsen, and J.-S. Wang, Phys. Rev. Lett. 58, 86 (1987).

10. U. Wolff, Phys. Rev. Lett. 62, 361 (1989).

11. B. Widom, J. Chem. Phys. 43, 3892 (1965).

12. K. G. Wilson, and J. Kogut, Phys. Rep. 12, 75 (1974).

13. L. D. Landau, Phys. Z. Sowjetunion 11, 26 (1937).

14. L. D. Landau, Zh. Eksp. Teor. Fiz. 7, 19 (1937).

15. L. D. Landau, Phys. Z. Sowjetunion 11, 545 (1937).

16. L. D. Landau, Zh. Eksp. Teor. Fiz. 7, 627 (1937).

17. F. Alet, A. M. Walczak, and M. P. A. Fisher, Physica A 369, 122 (2006).

18. S. Sachdev (2009), arXiv: 0901.4103.

19. D. Bitko, T. F. Rosenbaum, and G. Aeppli, Phys. Rev. Lett. 77, 940 (1996).

20. L. Demkó, S. Bordács, T. Vojta, D. Nozadze, F. Hrahsheh, C. Svoboda, B. Dóra, H. Yamada, M. Kawasaki, Y. Tokura, and I. Kézsmárki, Phys. Rev. Lett. 108, 185701 (2012).

21. M. Greiner, O. Mandel, T. Esslinger, T. W. Hänsch, and I. Bloch, Nature 415, 39 (2002).

22. H. F. Trotter, Proc. American Math. Soc. 10, 545 (1959).

23. R. P. Feynman, and A. R. Hibbs, Quantum Mechanics and Path Integrals, McGraw-Hill, New York, 1965.

24. S. L. Sondhi, S. M. Girvin, J. P. Carini, and D. Shahar, Rev. Mod. Phys. 69, 315 (1997).

25. M. N. Barber, "Finite-Size Scaling," in Phase Transitions and Critical Phenomena, edited by C. Domb, and J. L. Lebowitz, Academic, New York, 1983, vol. 8, pp. 145-266.

26. T. Vojta, D. Belitz, R. Narayanan, and T. R. Kirkpatrick, Europhys. Lett. 36, 191 (1996).

27. D. Belitz, T. R. Kirkpatrick, and T. Vojta, Phys. Rev. B 65, 165112 (2002).

28. D. Belitz, T. R. Kirkpatrick, and T. Vojta, Rev. Mod. Phys. 77, 579 (2005).

29. S. F. Edwards, and P. W. Anderson, Journal of Physics F: Metal Physics 5, 965 (1975).

30. K. H. Fischer, and J. A. Hertz, Spin glasses, Cambridge University Press, Cambridge, 1991.

31. A. B. Harris, J. Phys. C 7, 1671 (1974).

32. A. Weinrib, and B. I. Halperin, Phys. Rev. B 27, 413-427 (1983).

33. J. T. Chayes, L. Chayes, D. S. Fisher, and T. Spencer, Phys. Rev. Lett. 57, 2999 (1986).

34. Y. Imry, and S.-k. Ma, Phys. Rev. Lett. 35, 1399-1401 (1975).

35. M. Aizenman, and J. Wehr, Phys. Rev. Lett. 62, 2503 (1989).

36. T. Nattermann, J. Phys. C 16, 6407 (1983).

37. Y. Imry, and M. Wortis, Phys. Rev. B 19, 3580 (1979).

38. K. Hui, and A. N. Berker, Phys. Rev. Lett. 62, 2507 (1989). 
39. R. L. Greenblatt, M. Aizenman, and J. L. Lebowitz, Phys. Rev. Lett. 103, 197201 (2009).

40. P. Pfeuty, Phys. Lett. A 72, 245 (1979).

41. R. Narayanan, T. Vojta, D. Belitz, and T. R. Kirkpatrick, Phys. Rev. Lett. 82, 5132 (1999).

42. R. Narayanan, T. Vojta, D. Belitz, and T. R. Kirkpatrick, Phys. Rev. B 60, 10150 (1999).

43. S. K. Ma, C. Dasgupta, and C. K. Hu, Phys. Rev. Lett. 43, 1434 (1979).

44. D. S. Fisher, Phys. Rev. Lett. 69, 534 (1992).

45. D. S. Fisher, Phys. Rev. B 50, 3799 (1994).

46. D. S. Fisher, Phys. Rev. B 51, 6411 (1995).

47. R. B. Griffiths, Phys. Rev. Lett. 23, 17 (1969).

48. M. Wortis, Phys. Rev. B 10, 4665 (1974).

49. A. B. Harris, Phys. Rev. B 12, 203 (1975).

50. Y. Imry, Phys. Rev. B 15, 4448 (1977).

51. A. J. Bray, and D. Huifang, Phys. Rev. B 40, 6980 (1989).

52. P. C. Hohenberg, and B. I. Halperin, Rev. Mod. Phys. 49, 435 (1977).

53. R. J. Glauber, J. Math. Phys. 4, 294 (1963).

54. N. Metropolis, A. Rosenbluth, M. Rosenbluth, and A. Teller, J. Chem. Phys. 21, 1087 (1953).

55. D. Dhar, "Stochastic evolution in Ising models," in Stochastic Processes: Formalism and Applications, edited by D. S. Argawal, and S. Dattagupta, Springer, Berlin, 1983, p. 300.

56. M. Randeria, J. P. Sethna, and R. G. Palmer, Phys. Rev. Lett. 54, 1321 (1985).

57. A. J. Bray, Phys. Rev. Lett. 60, 720 (1988).

58. A. J. Bray, J. Phys. A. 22, L81 (1989).

59. T. Senthil, and S. Sachdev, Phys. Rev. Lett. 77, 5292 (1996).

60. T. Vojta, and J. A. Hoyos, "Quantum phase transitions on percolating lattices," in Recent Progress in Many-Body Theories, edited by J. Boronat, G. Astrakharchik, and F. Mazzanti, World Scientific, Singapore, 2008, p. 235.

61. B. M. McCoy, and T. T. Wu, Phys. Rev. Lett. 21, 549 (1968).

62. B. M. McCoy, and T. T. Wu, Phys. Rev. 176, 631 (1968).

63. B. M. McCoy, and T. T. Wu, Phys. Rev. 188, 982 (1969).

64. M. Thill, and D. A. Huse, Physica A 214, 321 (1995).

65. M. Guo, R. N. Bhatt, and D. A. Huse, Phys. Rev. B 54, 3336 (1996).

66. H. Rieger, and A. P. Young, Phys. Rev. B 54, 3328 (1996).

67. A. P. Young, and H. Rieger, Phys. Rev. B 53, 8486 (1996).

68. T. Vojta, and J. Schmalian, Phys. Rev. B 72, 045438 (2005).

69. H. G. Ballesteros, L. A. Fernandez, V. Martin-Mayor, A. Munoz Sudupe, G. Parisi, and J. J. RuizLorenzo, Phys. Rev. B 58, 2740 (1998).

70. R. Sknepnek, T. Vojta, and M. Vojta, Phys. Rev. Lett. 93, 097201 (2004).

71. J. Hooyberghs, F. Igloi, and C. Vanderzande, Phys. Rev. Lett. 90, 100601 (2003).

72. T. Vojta, J. Phys. A 36, 10921 (2003).

73. T. Vojta, Phys. Rev. Lett. 90, 107202 (2003).

74. T. Vojta, Phys. Rev. E 70, 026108 (2004).

75. M. Marcellini, M. Pärnaste, B. Hjörvarsson, and M. Wolff, Phys. Rev. B 79, 144426 (2009).

76. I. M. Lifshitz, Usp. Fiz. Nauk 83, 617 (1964), [Sov. Phys.-Usp. 7, 549 (1965)].

77. I. M. Lifshitz, Adv. Phys. 13, 483 (1964).

78. B. I. Halperin, and M. Lax, Phys. Rev. 148, 722 (1966).

79. R. Sknepnek, and T. Vojta, Phys. Rev. B 69, 174410 (2004).

80. F. Hrahsheh, D. Nozadze, and T. Vojta, Phys. Rev. B 83, 224402 (2011).

81. C. Svoboda, D. Nozadze, F. Hrahsheh, and T. Vojta, EPL (Europhysics Letters) 97, 20007 (2012).

82. G. Schehr, and H. Rieger, Phys. Rev. Lett. 96, 227201 (2006).

83. G. Schehr, and H. Rieger, J. Stat. Mech. 2008, P04012 (2008).

84. J. A. Hoyos, and T. Vojta, Phys. Rev. Lett. 100, 240601 (2008).

85. J. A. Hoyos, and T. Vojta, Phys. Rev. B 85, 174403 (2012).

86. A. O. Caldeira, and A. J. Legett, Ann. Phys. (N.Y.) 149, 374 (1983).

87. A. J. Leggett, S. Chakravarty, A. T. Dorsey, M. P. A. Fisher, A. Garg, and W. Zwerger, Rev. Mod. Phys. 59, 1 (1987).

88. U. Weiss, Quantum disspative systems, World Scientific, Singapore, 1993.

89. J. A. Hoyos, and T. Vojta, Phys. Rev. B 74, 140401(R) (2006). 
90. J. Hertz, Phys. Rev. B 14, 1165 (1976).

91. A. J. Millis, Phys. Rev. B 48, 7183 (1993).

92. H. von Löhneysen, A. Rosch, M. Vojta, and P. Wölfle, Rev. Mod. Phys. 79, 1015 (2007).

93. P. Gegenwart, Q. Si, and F. Steglich, Nature Physics 4, 186 (2008).

94. D. J. Thouless, Phys. Rev. 187, 732 (1969).

95. J. Cardy, J. Phys. A 14, 1407 (1981).

96. A. J. Millis, D. K. Morr, and J. Schmalian, Phys. Rev. Lett. 87, 167202 (2001).

97. A. J. Millis, D. K. Morr, and J. Schmalian, Phys. Rev. B 66, 174433 (2002).

98. A. H. Castro Neto, G. Castilla, and B. A. Jones, Phys. Rev. Lett. 81, 3531 (1998).

99. A. H. Castro Neto, and B. A. Jones, Phys. Rev. B 62, 14975 (2000).

100. G. S. Joyce, J. Phys. C: Solid State Phys. 2, 1531 (1969).

101. F. J. Dyson, Commun. Math. Phys. 12, 91 (1969).

102. P. Bruno, Phys. Rev. Lett. 87, 137203 (2001).

103. D. Nozadze, and T. Vojta, EPL (Europhysics Letters) 95, 57010 (2011).

104. J. A. Hoyos, C. Kotabage, and T. Vojta, Phys. Rev. Lett. 99, 230601 (2007).

105. T. Vojta, C. Kotabage, and J. A. Hoyos, Phys. Rev. B 79, 024401 (2009).

106. V. Dobrosavljevic, and E. Miranda, Phys. Rev. Lett. 94, 187203 (2005).

107. S. Guo, D. P. Young, R. T. Macaluso, D. A. Browne, N. L. Henderson, J. Y. Chan, L. Henry, and J. F. DiTusa, Phys. Rev. Lett. 100, 017209 (2007).

108. S. Guo, D. P. Young, R. T. Macaluso, D. A. Browne, N. L. Henderson, J. Y. Chan, L. L. Henry, and J. F. DiTusa, Phys. Rev. B 81, 144423 (2010).

109. S. Guo, D. P. Young, R. T. Macaluso, D. A. Browne, N. L. Henderson, J. Y. Chan, L. L. Henry, and J. F. DiTusa, Phys. Rev. B 81, 144424 (2010).

110. J. G. Sereni, T. Westerkamp, R. Küchler, N. Caroca-Canales, P. Gegenwart, and C. Geibel, Phys. Rev. B 75, 024432 (2007).

111. T. Westerkamp, M. Deppe, R. Küchler, M. Brando, C. Geibel, P. Gegenwart, A. P. Pikul, and F. Steglich, Phys. Rev. Lett. 102, 206404 (2009).

112. S. Ubaid-Kassis, T. Vojta, and A. Schroeder, Phys. Rev. Lett. 104, 066402 (2010).

113. A. Schroeder, S. Ubaid-Kassis, and T. Vojta, J. Phys. Condes. Matter 23, 094205 (2011).

114. D. Nozadze, and T. Vojta, Phys. Rev. B 85, 174202 (2012).

115. P. Mohan, R. Narayanan, and T. Vojta, Phys. Rev. B 81, 144407 (2010).

116. F. Hrahsheh, H. Barghathi, and T. Vojta, Phys. Rev. B 84, 184202 (2011).

117. P. Mohan, P. M. Goldbart, R. Narayanan, J. Toner, and T. Vojta, Phys. Rev. Lett. 105, 085301 (2010).

118. D. Pekker, G. Refael, and E. Demler, Phys. Rev. Lett. 105, 085302 (2010).

119. O. Motrunich, S. C. Mau, D. A. Huse, and D. S. Fisher, Phys. Rev. B 61, 1160 (2000).

120. R. M. Fernandes, and J. Schmalian, Phys. Rev. Lett. 106, 067004 (2011). 